\title{
Empirical Sensitivity of Two-Dimensional Nonlinear Wake-Cylinder Oscillators in Cross-Flow/In-Line Vortex-Induced Vibrations
}

\author{
Narakorn Srinil ${ }^{\dagger}$, Pierre-Adrien Opinel \& Francesca Tagliaferri \\ Newcastle University, School of Engineering, Newcastle upon Tyne, NE1 7RU, United Kingdom
}

\begin{abstract}
Phenomenological wake-cylinder oscillators have been extensively implemented for vortex-induced vibration (VIV) predictions. Although such models capture fundamental VIV phenomena, the maximum response estimations and comparisons with different experimental data reveal some quantitative discrepancies due to the model empiricism embedding some uncertainties through system variables. This vital issue has not been well addressed in the literature of VIV modelling. This paper presents a new comprehensive investigation into the sensitivity to empirical input variables of nonlinear wake-cylinder oscillators simulating the twodimensionally coupled cross-flow/in-line VIV and amplified mean displacements of a flexibly mounted circular cylinder in uniform flows. The fluid-structure coupling terms are advanced by accounting for the higher-order nonlinear effects of fluctuating lift-drag forces and steady-drag dynamic magnifications, depending on the relative flow-cylinder velocities. A random sampling and variance-based sensitivity studies are carried out using Monte Carlo simulations which are computationally efficient based on the reduced-order model. This enables a large series of parametric examinations. Individual contribution, relative importance, coupling and interdependence of multiple input variables affecting output uncertainties are qualitatively and quantitatively evaluated. The Reynolds number dependence is also captured by correlating the wake and hydrodynamic coefficients with experimental data. Parametric studies highlight greater variations in the predicted amplitudes and mean displacements of the cylinder two-degree-of-freedom VIV with a lower mass ratio. Numerical findings allow for the identification of a few most influential variables to be treated as the empirically tuned coefficients. The improved understanding of model versatility and sensitivity enhances the calibration confidence and the response predictability with a reduced computational effort.
\end{abstract}

Keywords: vortex-induced vibration (VIV), circular cylinder, empirical sensitivity, wake oscillator, output uncertainty, Reynolds number.

\footnotetext{
${ }^{\dagger}$ Corresponding author: narakorn.srinil@newcastle.ac.uk
} Tel. +44 191208 6499; Fax +44 1912085491 


\section{Nomenclature}

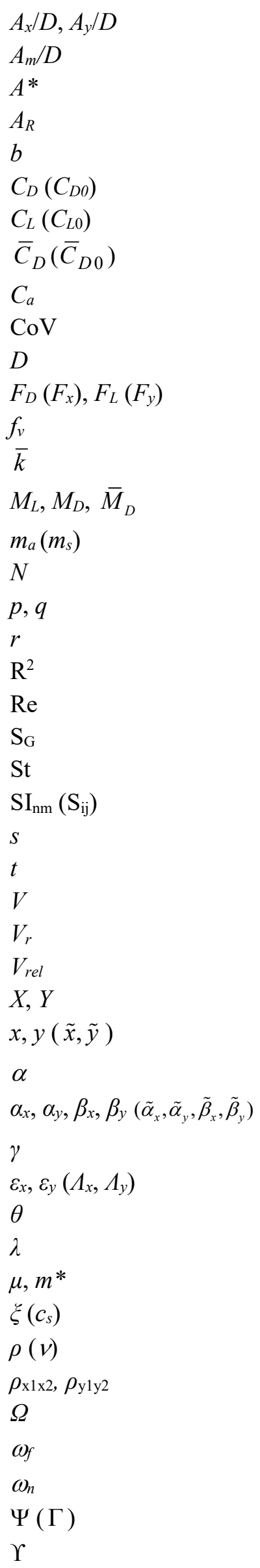

In-line and cross-flow amplitude per diameter In-line mean displacement per diameter Maximum cross-flow amplitude per diameter within a lock-in range Amplitude ratio between correlated and uncorrelated variable cases Regression analysis coefficients Unsteady drag force coefficient of oscillating (stationary) cylinder Unsteady lift force coefficient of oscillating (stationary) cylinder Mean drag force coefficient of oscillating (stationary) cylinder

Added mass coefficient of oscillating cylinder in still water Coefficient of variation

Cylinder outer diameter

Dimensional total drag (in-line) and lift (cross-flow) hydrodynamic forces Higher-order nonlinear effect of relative velocities

Tuning coefficient for mean drag amplification function

Combined hydrodynamic coefficient and mass ratio parameters

Fluid-added (cylinder) mass

Number of random values for each input variable

Wake oscillator variables modelling the fluctuating drag and lift forces

Residuals in the regression analysis

Coefficient of determination in the Pearson's correlation (PC) analysis

Reynolds number

Skop-Griffin parameter

Strouhal number

Sobol' (local) sensitivity indices

Normalising factor for standardized regression coefficients (SRC)

Dimensionless time

Free stream flow velocity

Nominal reduced flow velocity

Flow-cylinder relative velocities

In-line and cross-flow coordinates

Dimensionless (dimensional) in-line and cross-flow displacements

Mass-damping parameter

Geometrical nonlinearity coefficients in dimensionless (dimensional) form

Stall parameter

Empirical wake damping (wake-cylinder coupling) coefficients

Instantaneous angle of effective lift/drag forces acting on oscillating cylinder

Combined fluid-structural damping terms

Mass ratio expression

Structural damping ratio (coefficient)

Fluid density (kinematic viscosity)

Correlation coefficients of two variables used in Nataf Transformation

Ratio of vortex-shedding to cylinder cross-flow natural frequencies

Vortex-shedding angular frequency

Cross-flow angular natural frequency in still water

Vectors of output (input) variables in Monte Carlo simulations (MCS)

A set of input variables governing wake-cylinder oscillators 


\section{Introduction}

\subsection{Background}

Modelling of vortex-induced vibration (VIV) to estimate response amplitudes and capture the associated fluidstructure interaction phenomena has recently received great attention by the engineering and scientific research communities. These are driven primarily by a need from an offshore (oil \& gas, subsea, renewable wind/marine energy, aquaculture and mining) industry to improve the prediction of potentially serious effects of VIV on the structural integrity, safety and fatigue performances subject to ocean current flows. The operational downtime and fatigue failure caused by VIV and the improper implementation of VIV passive control devices can lead to substantial investments and costs. Notwithstanding the recent advances in VIV theories, semiempirical models and numerical simulations (Bearman, 2011; Sarpkaya, 2004; Williamson and Govardhan, 2004; Wu et al., 2012), a computationally efficient and reliable tool for VIV analysis and prediction involving several fluid-structure parameters and input variables are still needed from a practical design perspective.

Amongst a variety of numerical approaches, a phenomenological model based on the use of coupled wake-cylinder oscillators has undergone continuous improvement (Gabbai and Benaroya, 2005) due to a simple conception and minimal computational requirement in predicting a complex multi-degree-of-freedom VIV in a wide range of flow and structural parameters. There is a significant demand for such models as a full computational fluid dynamics simulation of VIV behaviours is not always possible or may be very demanding in typical practical analyses. In all wake-body oscillator models, an important connection between actual VIV physical phenomena and mathematical expressions is defined through multiple variables, coefficients or functions being treated as empirical input variables depending on calibration with experimental data. Owing to the interaction mechanisms of cross-flow and in-line VIV coupled with the dynamic magnification of inline mean displacements, the hydrodynamic and structural nonlinearities, and the effects of system parameters, a systematic investigation into the model empirical sensitivity is of theoretical and practical significance.

To the best of the authors' knowledge, a comprehensive investigation on empirical sensitivity of twodimensional nonlinear wake-body oscillators with multiple variable coefficients and parameters in simulating coupled cross-flow/in-line VIV and amplified mean displacements by also accounting for the influence of mass ratio and Reynolds number is still lacking in the literature. These insightful aspects will be herein studied through Monte Carlo simulations of the freely-oscillating circular cylinders in uniform flows.

\subsection{Wake-Cylinder Oscillators for VIV Prediction}

For VIV predictions of flexibly mounted circular cylinders, several researchers proposed and modified the coupled wake-cylinder oscillators since the pioneering idea of Bishop and Hassan (1964b) who suggested the potential use of a nonlinear oscillator to mimic a periodic alteration of vortex-shedding process. There are also some physical explanations of the fluid wake circulations linked to the oscillators (Blevins, 1990; Iwan and Blevins, 1974). A detailed theoretical discussion on the van der Pol wake oscillator in modelling the fluctuating lift force leading to a one-degree-of-freedom (1-DOF) cross-flow VIV was presented by Facchinetti et al. (2004). By considering a linear oscillator with mass, damping and stiffness for a rigid cylinder, the wake oscillator typically contains two empirical coefficients capturing the self-limiting character through the wake damping, and the fluid-solid interaction through the cylinder acceleration. Both coefficients have been treated 
by several researchers as a constant. An alternative 1-DOF wake oscillator model for cross-flow-only VIV was presented by Skop and Balasubramanian (1997) and Krenk and Nielsen (1999), who considered the cylinder velocity-based coupling term, and Ogink and Metrikine (2010) who introduced the frequency-dependent coupling term. The latter work discussed a key concern in VIV prediction with a single set of empirical coefficients to match different response branches with experimental data. This challenge becomes even more problematic when the cylinder in-line mean and oscillatory components are also accounted for, increasing the unknown DOFs, system input parameters and difficulty in tuning the empirical coefficients.

For the cylinder 2-DOF VIV subject to fluctuating lift and drag forces, Srinil and Zanganeh (2012) applied double van der Pol wake oscillators to further account for the interactions of cross-flow and in-line VIV through the cylinder's Duffing-type oscillators with cubic and quadratic nonlinearities. Essentially, the cubic geometric nonlinearities capture a hardening-type response observed in cylinder 2-DOF VIV experiments with a low mass ratio (Jauvtis and Williamson, 2004) whereas the quadratic nonlinearities simulate the wakecylinder coupling, being responsible for a dual 2:1 resonance and figure-of-eight appearance (Dahl et al., 2010; Srinil et al., 2013). By calibrating the model with experimental data of Stappenbelt et al. (2007) and Blevins and Coughran (2009), Srinil and Zanganeh (2012) proposed an empirical formula for the wake damping coefficient as a function of mass and/or damping ratio. Zanganeh and Srinil (2014) further improved their early model by correctly accounting for the relative velocities between the cylinder and the flow (Blevins, 1990; Paidoussis et al., 2011), and applying a harmonic balance to analytically characterize variable hydrodynamic lift/drag and added mass coefficients for the resonantly oscillating cylinders. Recently, such wake oscillators have been extended to a three-dimensional VIV analysis of long flexible cylinders with axial dynamics, mean drag amplifications and multi DOFs (Zanganeh and Srinil, 2016).

The wake oscillator model has recently been applied to other applications including energy harvesting (Franzini and Bunzel, 2018), flexible pipes carrying internal flows (Yang et al., 2018) and VIV in oscillatory flows (Opinel and Srinil, 2018). Nevertheless, an empirical sensitivity to justify the input versus output variabilities has not been studied in the aforesaid works. This aspect will be herein comprehensively addressed.

\subsection{Literature Related to Uncertainty in VIV Modelling}

Offshore fluid-structure interaction analyses and designs are governed by several input/output uncertainties (Bitner-Gregersen and Hagen, 1990). A few studies have investigated the effect of input uncertainty and its propagation in VIV simulation models. Xiu et al. (2002) applied a generalized polynomial chaos algorithm to model the stochastic flow-cylinder interaction due to cross-flow-only VIV. They treated the cylinder damping and spring stiffness coefficients as independent standard Gaussian random variables. By coupling the NavierStokes and cylinder equations, the flow domain is stochastic due to the cylinder random boundary condition influencing the flow fields. Numerical simulations showed the effect of such input randomness on both the fluid force and cylinder responses. A similar approach has been implemented by Lucor et al. (2003) who showed different predicted responses based on the stochastic vs. deterministic solutions. This random effect appears to be significant even though these computational fluid dynamics studies considered flows at a very low Reynolds number of 100 . For systems with high dimensionality, i.e. with several input variables as in the present study, Lucor et al. (2004) suggested using the Monte Carlo approach for random oscillators. 
For a multi-DOF riser, there has been some attempts to capture the VIV response prediction uncertainty. Based on a frequency domain approach, Lie et al. (2008) accounted for the stochastic VIV response of flexible risers by introducing a time varying envelope function combined with a time sharing between the dominating frequencies. This approach allows the combination of response standing and travelling waves and the improved fatigue estimation. Mukundan et al. (2010) developed an approach to reconstruct the riser spatial response from a limited number of measurement sensors located along its length, and identified three sources of uncertainties contributing to the reconstruction errors. These include the presence of noise in experimental data, the use of accelerometers vs. strain gauges for recording response motions, and the use of trigonometric terms during the reconstruction. Lie and Kaasen (2006) identified the riser curvature measurement errors by propagating the noise to the variability in the modal weighting factors used for reconstructing the riser response through a modal analysis. They showed that the low-order riser modes are very sensitive to the measurement noise. Recently, a stochastic modelling of cross-flow VIV in time domain has been presented by Ulveseter et al. (2017) who introduced a Gaussian random process through a synchronization between vortex shedding and cylinder frequencies. They predicted a chaotic VIV response depending on the specified empirical coefficients and the assumed random process spectrum.

A few published works have carried out a sensitivity analysis of VIV responses based on wake-cylinder oscillator models. Through Monte Carlo simulations, Gabbai and Hiebert (2011) quantified the relative importance of five independent input parameters in the 1-DOF cross-flow VIV model of Facchinetti et al. (2004). By considering a uniform distribution of each wake or cylinder parameter, numerical results suggested a greatest effect of the fluid forcing term in the cylinder equation of motion. For a vertical riser undergoing cross-flow VIV in uniform or sheared flows, Modarres-Sadeghi et al. (2008) considered the above wake oscillator model by randomly and independently varying the wake oscillator and hydrodynamic coefficients along the riser length in an attempt to account for the strong randomness observed in the associated experimental data. They showed that such input randomness leads to an improvement of riser fatigue calculation. More recently, Low and Srinil (2016) presented a systematic statistical approach to perform a fatigue reliability analysis of a vertical riser undergoing cross-flow VIV based on a wake oscillator proposed by Skop and Balasubramanian (1997). Up to seven random parameters were considered, including wake and hydrodynamic coefficients, current velocity, riser properties and S-N curve parameters. The interdependence effect of empirical input variables was also analysed using the Nataf Transformation. Their results showed that the randomness in wake coefficients leads to large variability in the riser fatigue damage estimation, and that the interdependence of coefficients should be also accounted for. Nevertheless, the in-line VIV modelling feature has been overlooked by the above wake oscillator-based studies. This will be herein considered based on the two-dimensional coupled nonlinear wake-body oscillators.

\subsection{Objectives}

The main objectives of this research are:

(i) To present the advanced nonlinear wake-cylinder oscillators and investigate the empirical sensitivity and relative input contributions to the output uncertainties in predicting coupled cross-flow and in-line VIV responses and the associated dynamic amplification of in-line mean displacements, 
(ii) To identify which input variables play the most influential role in VIV predictions and to demonstrate the interdependence effect of input variables, through several numerical means and parametric explorations,

(iii) To evaluate the influence of Reynolds number (Re) through the hydrodynamic and empirically-tuned coefficients by introducing some useful empirical functions incorporating available experimental data.

Ultimately, the analysis outcomes fulfilling these objectives will improve the model prediction capability with better understanding of empirical input variability and enhance the confidence in implementing industrial tools with wake oscillators being applied to the VIV analysis of rigid and flexible cylindrical structures.

This paper is structured as follows. In Section 2, the advanced wake-cylinder oscillators are presented, highlighting several key features and a selection of random input variables. In Section 3, methodologies for sensitivity analyses, including the Re effect, are explained. Parametric studies are carried out in Section 4 followed by some discussion remarks in Section 5. The paper ends with the conclusions in Section 6.

\section{Two-Dimensional Nonlinear Wake-Cylinder Oscillators}

A reduced-order fluid-structure interaction model, simulating coupled cross-flow/in-line VIV and in-line mean displacements, is presented and investigated. Figure 1 displays a two-dimensional spring idealisation of a flexibly mounted circular cylinder placed in a steady and uniform flow of velocity $(V)$. By accounting for the system relative velocities $\left(V_{r e l}\right)$ between the free stream flow and the two-DOF moving cylinder, a dynamic angle of attack $\theta$ (Blevins, 1990) may be realized such that the instantaneous directions of effective $\operatorname{drag}\left(F_{D}\right)$ and lift $\left(F_{L}\right)$ forces acting on the oscillating cylinder are aligned with and perpendicular to the $V_{\text {rel }}$ vector, respectively. Consequently, both $F_{D}$ and $F_{L}$ components can be projected onto the global in-line $(X)$ and crossflow $(Y)$ axes, enabling the overall hydrodynamic drag-lift coupling as discussed in Ogink and Metrikine (2010) and Zanganeh and Srinil (2016).

\subsection{System Equations and Variables}

Recent 2-DOF VIV experiments reported large-amplitude oscillations and amplified mean displacements for the two-dimensional spring-cylinder system placed in uniform flows (Srinil et al., 2018). For the vibration model drawn in Fig. 1, the dynamic equilibrium of cylinder motions may be derived and simplified as in the Appendix. The geometrically nonlinear cubic-type oscillators, accounting for the intrinsic two-dimensional displacement coupling and stretching effects, may be expressed in a dimensional form for cylinder in-line and cross-flow VIV, with the bi-directionally equal quantities of structural mass $\left(m_{s}\right)$, fluid-added mass $\left(m_{a}\right)$, structural viscous damping $\left(c_{s}\right)$ and linear stiffness coefficient $(K)$, as

$$
\begin{aligned}
& \left(m_{s}+m_{a}\right) \ddot{\tilde{x}}+c_{s} \dot{\tilde{x}}+K\left(\tilde{x}+\tilde{\alpha}_{x} \tilde{x}^{3}+\tilde{\beta}_{x} \tilde{x} \tilde{y}^{2}\right)=F_{D} \cos \theta-F_{L} \sin \theta, \\
& \left(m_{s}+m_{a}\right) \ddot{\tilde{y}}+c_{s} \dot{\tilde{y}}+K\left(\tilde{y}+\tilde{\alpha}_{y} \tilde{y}^{3}+\tilde{\beta}_{y} \tilde{y} \tilde{x}^{2}\right)=F_{D} \sin \theta+F_{L} \cos \theta,
\end{aligned}
$$

where, as in Fig.1, $\sin \theta=-\dot{\tilde{y}} / V_{\text {rel }}$ and $\cos \theta=(V-\dot{\tilde{x}}) / V_{\text {rel }}$. A dot denotes differentiation with respect to time, $\tilde{x}$ and $\tilde{y}$ are the cylinder in-line and cross-flow displacements, $\tilde{\alpha}_{x}, \tilde{\beta}_{x}, \tilde{\alpha}_{y}$ and $\tilde{\beta}_{y}$ are the geometric nonlinearity coefficients, $m_{a}=\rho \pi D^{2} C_{a} / 4, \rho$ the fluid density, $D$ the cylinder diameter, $C_{a}$ the added mass coefficient. Following Zanganeh and Srinil (2016), the total drag and lift forces per unit length and $V_{r e l}$ are expressed as 


$$
\begin{gathered}
F_{D}=\frac{1}{2} \rho D V_{r e l}^{2}\left(\bar{C}_{D}+C_{D}\right), \quad F_{L}=\frac{1}{2} \rho D V_{r e l}^{2} C_{L}, \\
V_{r e l}=V \sqrt{\left(1-\frac{\dot{\tilde{x}}}{V}\right)^{2}+\left(\frac{\dot{\tilde{y}}}{V}\right)^{2}},
\end{gathered}
$$

in which the mean drag, fluctuating drag and lift force coefficients of the oscillating cylinder are defined by $\bar{C}_{D}, C_{D}$ and $C_{L}$, whereas their counterparts for the stationary cylinder are $\bar{C}_{D 0}, C_{D 0}$ and $C_{L 0}$, respectively.

To simulate the modification of stationary fluid forces due to the wake vortex shedding of the oscillating cylinder, variations in $C_{D}$ and $C_{L}$ are considered through the normalized wake variables as $C_{D}=C_{D 0} / 2$ (Srinil and Zanganeh, 2012) and $C_{L}=C_{L 0} q / 2$ (Facchinetti et al., 2004). These terms are substituted into Eq. (3) governing Eqs. (1) and (2). To capture the self-excitation and -limiting phenomena of cylinder VIV, the fluctuation of $p$ and $q$ may be described through the nonlinear van der Pol wake oscillators (Gabbai and Benaroya, 2005). Accordingly, by introducing the dimensionless time $(t)$ normalized with respect to the crossflow angular natural frequency in still water $\left(\omega_{n}\right)$, and the dimensionless displacements $x=\tilde{x} / D$ and $y=\tilde{y} / D$, Eqs. (1) and (2) can be transformed into the dimensionless forms, with four output variables $(x, p, y, q)$, as

$$
\begin{aligned}
& \ddot{x}+\lambda(\dot{x}, \dot{y}) \dot{x}+x+\alpha_{x} x^{3}+\beta_{x} x y^{2}=\left\{M_{D} p+2 \pi M_{L}\left(\frac{q \dot{y}}{V_{r}}\right)+\bar{M}_{D}-2 \pi M_{D}\left(\frac{p \dot{x}}{V_{r}}\right)\right\} \Omega^{2} f_{V}(\dot{x}, \dot{y}), \\
& \ddot{p}+2 \varepsilon_{x} \Omega\left(p^{2}-1\right) \dot{p}+4 \Omega^{2} p=\Lambda_{x} \ddot{x}, \\
& \ddot{y}+\lambda(\dot{x}, \dot{y}) \dot{y}+y+\alpha_{y} y^{3}+\beta_{y} y x^{2}=\left\{M_{L} q-2 \pi M_{D}\left(\frac{p \dot{y}}{V_{r}}\right)-2 \pi M_{L}\left(\frac{q \dot{x}}{V_{r}}\right)\right\} \Omega^{2} f_{V}(\dot{x}, \dot{y}), \\
& \ddot{q}+\varepsilon_{y} \Omega\left(q^{2}-1\right) \dot{q}+\Omega^{2} q=\Lambda_{y} \ddot{y},
\end{aligned}
$$

in which $\varepsilon_{x}, \Lambda_{x}, \varepsilon_{y}$ and $\Lambda_{y}$ are the empirical wake coefficients regulating the self-limiting response $\left(\varepsilon_{x}, \varepsilon_{y}\right)$ and the fluid-cylinder interaction $\left(\Lambda_{x}, \Lambda_{y}\right), \alpha_{x}, \beta_{x}, \alpha_{y}$ and $\beta_{y}$ are the geometrically nonlinear coefficients, and $V_{r}$ is the nominal reduced flow velocity $V_{r}=2 \pi V / \omega_{n} D$, with the normalized fluid excitation frequency $\Omega=\operatorname{St} V_{r}=\omega_{f} / \omega_{n}$ and the angular vortex-shedding frequency $\omega_{f}$ according to the Strouhal number (St) rule. With equal $X$-Y natural frequencies, the dimensionless quantities $\left(M_{L}, M_{D}, \bar{M}_{D}\right)$, the higher-order nonlinear effect of relative velocities $f_{v}(\dot{x}, \dot{y})$, the system damping $(\lambda)$ and the mass relationship $(\mu)$ can be expressed, respectively, as

$$
\begin{gathered}
M_{L}=\frac{C_{L 0}}{16 \pi^{2} \mathrm{St}^{2} \mu}, \quad M_{D}=\frac{C_{D 0}}{16 \pi^{2} \mathrm{St}^{2} \mu}, \quad \bar{M}_{D}=\frac{\bar{C}_{D}}{8 \pi^{2} \mathrm{St}^{2} \mu}, \\
f_{V}(\dot{x}, \dot{y})=\sqrt{\left(1-2 \pi \frac{\dot{x}}{V_{r}}\right)^{2}+\left(2 \pi \frac{\dot{y}}{V_{r}}\right)^{2}}, \\
\lambda=2 \xi+\left(\frac{\bar{C}_{D} V_{r}}{4 \pi \mu}\right) f_{V}(\dot{x}, \dot{y}), \\
\mu=\frac{\left(m_{s}+m_{a}\right)}{\rho D^{2}}=\frac{\pi}{4}\left(m^{*}+C_{a}\right),
\end{gathered}
$$

where $\xi$ is the structural damping ratio and $m^{*}$ is the mass ratio with $m^{*}=m_{s} /\left(\rho \pi D^{2} / 4\right)$. The coefficient $C_{a}$ may be assumed to be unity for a circular cylinder oscillating in still water (Blevins, 1990). Nevertheless, due to the unsteady wake dynamics, the effective added mass is time-dependent which is already accounted for 
through the wake oscillators as analytically characterized in Zanganeh and Srinil (2014) by decomposing the total hydrodynamic forces in Eqs. (5) and (7) to be in phase with the cylinder accelerations.

A linearization with $V_{r e l} \approx V, \sin \theta \approx \dot{y} / V$ and $\cos \theta \approx 1$ may be further applied to eliminate nonlinear terms $p \dot{x}, q \dot{x}$ and $f_{v}$ from Eqs. (5) and (7) as in Zanganeh and Srinil (2014) who also disregarded $\bar{M}_{D}$ in order to focus on the oscillation components. In this study, the full nonlinear expressions of Eqs. (5) and (7) are considered as a more complete and generic model. Numerical integrations of Eqs. (5)-(8) can be performed by using the Runge-Kutta solver in Matlab with adaptive time steps and appropriate initial conditions. In each simulation case, the final steady-state responses of about 100 cycles of oscillations are kept and statistically analysed.

\subsection{Summary of Phenomenological Aspects}

Some key features captured by the present nonlinear wake-body oscillators are highlighted as follows.

a) Dual 2:1 Resonance: The $X-Y$ response trajectories exhibit various figure-of-eight patterns, $X-Y$ phase differences and tuned 2:1 oscillation frequencies due to the adapted hydrodynamic added mass (Dahl et al., 2010). In accordance with the 2:1 drag-lift vortex-shedding frequency ratio, such a dual resonance character is associated with quadratic nonlinearities (Vandiver and Jong, 1987) and can be captured through the wakecylinder coupling ( $q \dot{y}, p \dot{x}, p \dot{y}, q \dot{x})$ terms (Zanganeh and Srinil, 2014) in Eqs. (5) and (7).

b) Self-Limiting Feature: Both structural viscous (linear) and hydrodynamic (nonlinear) damping effects are accounted for through Eq. (11). The time- and amplitude-dependent hydrodynamic damping strongly depends on the relative velocities in both $X-Y$ directions $\left(f_{v}\right)$ as well as on $\bar{C}_{D}$. The $\bar{C}_{D} V_{r} / 4 \pi \mu$ expression is equivalent to $\gamma \Omega / \mu$ as in Srinil and Zanganeh (2012) with $\gamma=\bar{C}_{D} / 4 \pi \mathrm{St}$ being the so-called stall parameter (Skop and Balasubramanian, 1997). If a constant $\gamma$ is assigned, e.g. $\gamma=0.8\left(\bar{C}_{D} \approx 2, \mathrm{St}=0.2\right)$ as in Facchinetti et al. (2004), the dynamic variation of $\bar{C}_{D}$ is omitted. In the absence of $\xi$, the stall term solely contributes to the cylinder self-limiting or limit cycle feature.

c) Mean Drag Amplification: Physical experiments have evidenced that the steady or mean drag force can be dynamically and significantly amplified during the VIV lock-in or synchronization for the oscillating cylinder. The magnification of $\bar{C}_{D}$ may be described as a linear function of maximum cross-flow amplitude per diameter $\left(A^{*}\right)$ within a certain lock-in range. A typical empirical expression reads (Blevins, 1990)

$$
\bar{C}_{D}=\bar{C}_{D 0}\left(1+\bar{k} A^{*}\right)
$$

where $\bar{k}$ is an empirical factor which may be calibrated with 1-DOF (Blevins, 1990) or 2-DOF VIV (Stappenbelt et al., 2007) data. $A^{*}$ is unknown and may be specified a priori based on an existing correlation in the literature. This point will be again discussed in Section 3.2 together with the Re effect.

d) Cylinder In-Line Drift: The $\bar{M}_{D}$ term multiplied with $f_{v}$ in Eq. (5) has two effects on the cylinder. The first one results in an in-line static drift due to the stationary mean drag force component proportional to $V^{2}$ whereas the second one results in the mean drag dynamic amplification depending on oscillation amplitudes. These drifts admit positive values in the $X$ direction. In addition to $\bar{M}_{D}$, smaller drifts due to 2:1 resonances are also generated by the quadratic $(q \dot{y})$ and cubic $\left(x y^{2}\right)$ nonlinear terms as theoretically proved in Zanganeh 
and Srinil (2014). These extra values may be positive or negative depending on the relative $(q-y$ or $x-y)$ phases. Nevertheless, a combination of overall drift components is found to always yield a positive value suggesting that a final new equilibrium of the displaced cylinder will take place further downstream.

e) Empiricism: Overall, the present reduced-order model encompasses 12 empirical input variables classified into 3 groups as follows:

(i) Wake oscillator coefficients $\left(\varepsilon_{x}, \Lambda_{x}, \varepsilon_{y}, \Lambda_{y}\right)$,

(ii) Cylinder geometric nonlinearity coefficients $\left(\alpha_{x}, \beta_{x}, \alpha_{y}, \beta_{y}\right)$, and

(iii) Hydrodynamic coefficients (St, $\left.C_{D 0}, C_{L 0}, \bar{C}_{D}\right)$.

Based on calibration with experimental 2-DOF VIV data (Blevins and Coughran, 2009; Stappenbelt et al., 2007), $\varepsilon_{y}$ may be assumed through the $m^{*}$-based function (Zanganeh and Srinil, 2014):

$$
\varepsilon_{y}=0.00234 e^{\left(0.228 m^{*}\right)} .
$$

As the dynamic amplification of $\bar{C}_{D}$ may be based Eq. (13) which contains another empirical variable $\bar{k}$ and unknown $A^{*}$. Without specifying these two input variables a priori, Eqs. (5)-(8) would be solved by iterations which are very time-consuming and not recommended. To overcome this difficulty, $\bar{k}$ and $A^{*}$ will be specified based on calibration with experimental data as in Sections 3.2 and 4.3, rendering a total of 14 model empiricisms. In the following, the process of selecting the random input variables is described.

\subsection{Identification of Random Input Variables}

Table 1 summarizes the dimensionless quantities of hydrodynamic coefficients and fluid-structure parameters appearing in Eqs. (5)-(13). It is noticed that most of the quantities depend on $m^{*}$ which is one the key parameters in 2-DOF VIV (Jauvtis and Williamson, 2004). $\xi$ may also contain some uncertainties (Low and Srinil, 2016); however, a small $\xi$ (order of $10^{-3}$ ) is herein specified as attention is placed on the $m^{*}$ effect on empirical sensitivity. To identify a set of influential coefficients to be considered as random input variables in Sections 3 and 4, a differential or local sensitivity analysis is first performed by applying one-at-a-time variations around the nominal values summarized in Table 2 for the above-mentioned 12 empirical coefficients. A perturbation to their nominal values is considered, and simulations with nominal values are used as a baseline case. Herein, the local sensitivity indices $\left(S_{\mathrm{ij}}\right)$ proportional to the derivatives of output variables $\left(\Psi_{\mathrm{j}}, \mathrm{j}=1-3\right)$ with respect to the chosen input variables $\left(\Upsilon_{i}, \mathrm{i}=1-12\right)$ are evaluated using a finite difference method. With the first-order forward difference of $\Psi_{j}$, the numerical approximation for $S_{i j}=\Upsilon_{i}\left[\Psi_{j}\left(\Upsilon_{i}+\Delta \Upsilon_{i}\right)-\Psi_{j}\left(\Upsilon_{i}\right)\right] / \Delta \Upsilon_{i}$, where $\Psi_{1}, \Psi_{2}$ and $\Psi_{3}$ denote cross-flow amplitude $\left(A_{y} / D\right)$, in-line amplitude $\left(A_{x} / D\right)$ and in-line mean displacement $\left(A_{m} / D\right)$, respectively. As $\mathrm{S}_{\mathrm{ij}}$ is based on a local linearity assumption, a small perturbation $\Delta \Upsilon_{\mathrm{i}}$ of $5 \%$ is applied to $\Upsilon_{i}$, indicating a reduced parameter space. A linear differential approximation may be invalid for a larger perturbation, see, e.g., Cattarin et al. (2018). To overcome this shortcoming, a global sensitivity analysis should instead be considered as in Sections 3 and 4.

Numerical results of $\mathrm{S}_{\mathrm{ij}}$ in the case of varying $V_{r}$ are illustrated in Fig. 2 based on $m^{*}=3.68$ and $\xi=0.006$, distinguishing between the set of wake-cylinder (a-c) and hydrodynamic (d-f) coefficients for the three main outputs. It can be noticed that the cross-flow wake coefficient $\varepsilon_{y}$ has a greatest influence on all output 
predictions, especially near the maximum response at $V_{r} \approx 10$. Other oscillator variables including $\Lambda_{y}, \alpha_{x}, \beta_{x}$, $\alpha_{y}$ and $\beta_{y}$ also play a role in the predicted $A_{y} / D, A_{x} / D$ or $A_{m} / D$, albeit with a lesser effect than $\varepsilon_{y}$. Nevertheless, a variation in $\varepsilon_{x}$ and $\Lambda_{x}$ is negligible, confirming what has been remarked in Srinil and Zanganeh (2012) based on a linearized model predicting only $A_{y} / D$ and $A_{x} / D$. As for the hydrodynamic coefficients, the greatest effect comes from $C_{L 0} . \bar{C}_{D}$ also plays an influential role in the associated $A_{m} / D$ prediction whereas St shows a small sensitivity effect. The $C_{D 0}$ variation is seen to be negligible: this is consistent with the above-observed trivial effect of $\varepsilon_{x}$ and $\Lambda_{x}$ variations governing the in-line wake oscillator with a smaller-amplitude excitation.

In the following, the sampling- and variance-based global sensitivity approaches are described by omitting $\varepsilon_{x}$ and $\Lambda_{x}$ variations and accounting for a greater parameter space with multiple random variables of $\varepsilon_{y}, \Lambda_{y}, \alpha_{x}$, $\beta_{x}, \alpha_{y}$ and $\beta_{y}$ (Section 3.1). Randomness in St, $C_{D 0}, C_{L 0}$ and $\bar{C}_{D}$ is also considered based on Re variations.

\section{Global Sensitivity Analysis: Qualitative and Quantitative Methodologies}

In most studies that use wake-cylinder oscillators, very little emphasis is placed on how a multitude of input variables present in the governing equations affects VIV predictions. In addition, no information on individual contribution, relative importance and coupling of variables is available. These aspects will be qualitatively and quantitatively measured through a global sensitivity by applying the Monte Carlo simulation (MCS) to assess the 2-DOF VIV multiple times under randomly selected inputs. By apportioning the output uncertainties to the input variability, the measured cylinder responses are cross-flow amplitudes $\left(A_{y} / D\right)$, in-line amplitudes $\left(A_{x} / D\right)$ and in-line mean displacements $\left(A_{m} / D\right)$. The $X$-Y oscillation frequencies are not treated as uncertain outputs as most of the $X-Y$ trajectories exhibit the figure-of-eight patterns due to 2:1 resonances regardless of the inputs.

\subsection{Sensitivity to Wake-Cylinder Oscillator Variables}

The six wake-cylinder oscillator variables are first considered and grouped as $\Upsilon=\left(\alpha_{x}, \alpha_{y}, \beta_{x}, \beta_{y}, \varepsilon_{y}, \Lambda_{y}\right)$. Their nominal values in Table 2 are treated as mean values in MCS, unless stated otherwise. A uniform distribution over an interval centred on such mean values of $\Upsilon$ is introduced as no associated information on individual and joint distributions is available. The use of uniform distribution is plausible since a sensitivity analysis depends more on the range than on the assigned distribution (Saltelli et al., 2000). Herein, a random sampling function in Matlab is used to create 6 vectors, each containing $N$ independent random numbers between 0.8 and 1.2 to allow for the $\pm 20 \%$ variation in $\Upsilon$. A variation of $10 \%$ was considered by Gabbai and Hiebert (2011) for a 1DOF VIV analysis. This increased $\pm 20 \%$ variation is due to the doubled dimensionality and 2-DOF coupling effect. Such sampling technique has an advantage in producing unbiased estimates of the output mean and variance (Saltelli et al., 2000). Subsequently, 6 series of $N$ perturbed values are generated by multiplying the mean values of $\Upsilon$ with the corresponding $N$ random numbers. By keeping the hydrodynamic coefficients in Table 2 invariant, Eqs. (5)-(8) are evaluated $N$ times, and the recorded steady-state $X-Y$ time histories are transformed into multiple $A_{y} / D, A_{x} / D$ and $A_{m} / D$ outputs for which the uncertainty analysis can be performed by evaluating the relevant mean and variance.

Both qualitative and quantitative characterizations of response outcomes can be achieved through several methods. The most convenient qualitative approach is the scatterplots or data cloud relating outputs $\left(A_{y} / D\right.$, 
$\left.A_{x} / D, A_{m} / D\right)$ to the multivariate random inputs $(\Upsilon)$. These scatterplots allow for a visual inspection of potential dependencies, being regular or complex, and the subsequent use of appropriate quantitative methods. As the obtained data cloud presented in Section 4.1 mostly display a linear relationship, the sensitivity quantification can be first performed by evaluating the Pearson's correlation coefficients (PC). By letting each output vector be $\Psi_{i}(i=1, \ldots, N)$ and the random input variables be $\Gamma_{i j}(j=1, \ldots, 6)$ for each input vector $\Upsilon_{j}\left(\Upsilon_{1}=\alpha_{x}, \Upsilon_{2}=\alpha_{y}\right.$, $\Upsilon_{3}=\beta_{x}, \Upsilon_{4}=\beta_{y}, \Upsilon_{5}=\varepsilon_{y}, \Upsilon_{6}=\Lambda_{y}$ ), the PC for each $\Upsilon_{j}$ can be evaluated through

$$
\begin{gathered}
\mathrm{PC}_{j}=\frac{\sum_{i=1}^{N}\left(\Gamma_{i j}-\bar{\Gamma}_{j}\right)\left(\Psi_{i}-\bar{\Psi}\right)}{\sqrt{\left[\sum_{i=1}^{N}\left(\Gamma_{i j}-\bar{\Gamma}_{j}\right)^{2}\right]} \sqrt{\left[\sum_{i=1}^{N}\left(\Psi_{i}-\bar{\Psi}\right)^{2}\right]}}, \\
\bar{\Psi}=\sum_{i=1}^{N} \frac{\Psi_{i}}{N}, \quad \bar{\Gamma}_{j}=\sum_{i=1}^{N} \frac{\Gamma_{i j}}{N} .
\end{gathered}
$$

Additional useful quantitative measure based on the regression analysis is also performed to identify contributions of individual inputs to the output uncertainties. With the $6 \mathrm{~N}$ dimensional random sampling strategy, a linear regression model for each output takes the form of

$$
\Psi_{i}=b_{0}+\sum_{j=1}^{6} b_{j} \Gamma_{i j}+r_{i}
$$

where $b_{j}$ are the regression coefficients and $r_{i}$ is the residual due to the approximation. These $b_{j}$ and $r_{i}$ values can be obtained through the least-squares approximation (Draper and Smith, 1981) directly applied to the scatter maps. For identifying and comparing the relative importance of each input variables, the standardized regression coefficients (SRC) are used for which $\mathrm{SRC}=b_{j} \tilde{s}_{j} / \tilde{s}$, where

$$
\tilde{s}=\left[\sum_{i=1}^{N} \frac{\left(\Psi_{i}-\bar{\Psi}\right)^{2}}{N-1}\right]^{1 / 2}, \quad \tilde{S}_{j}=\left[\sum_{i=1}^{N} \frac{\left(\Gamma_{i j}-\bar{\Gamma}_{j}\right)^{2}}{N-1}\right]^{1 / 2} .
$$

Eq. (18) implies that estimating SRC is equivalent to performing the regression analysis with input/output variables normalised to a zero mean and standard deviation of one. The associated coefficients of determination $\left(\mathrm{R}^{2}\right)$, which justify how reliably Eq. (17) can reproduce the actual outputs, are also computed through

$$
\mathrm{R}^{2}=\left[\sum_{i=1}^{N}\left(\tilde{\Psi}_{i}-\bar{\Psi}\right)^{2}\right] /\left[\sum_{i=1}^{N}\left(\Psi_{i}-\bar{\Psi}\right)^{2}\right]
$$

where $\tilde{\Psi}_{i}$ denotes the estimation of $\Psi_{i}$ obtained from Eq. (17). It should be noted that the regression model will achieve the best performance when $\mathrm{R}^{2}=1$ (Saltelli et al., 2000).

To also elaborate the multivariable interaction or the coupling effect of input changes on the predicted cross-flow/in-line VIV, the so-called Sobol' sensitivity indices (SI) are considered in conjunction with MCS (Sobol, 2001). In supporting the above quantitative observation of individual input contributions using SRC and $\mathrm{R}^{2}$, the interaction between each two independently random variables (e.g., within the wake oscillator, within the cylinder oscillator or cross-coupling between wake/cylinder oscillators) is herein of primary interest. According to the variance-based theory (Saltelli et al., 2000), the second-order $\mathrm{SI}_{n m}$ - which capture the model sensitivity to both $\Gamma_{n}$ and $\Gamma_{m}$ changes - are evaluated through 


$$
\begin{gathered}
\mathrm{SI}_{n m}=\frac{\tilde{V}\left(E\left(\Psi \mid \Gamma_{n}, \Gamma_{m}\right)\right)-\tilde{V}_{n}-\tilde{V}_{m}}{\tilde{V}(\Psi)}, \\
\tilde{V}_{n}=\tilde{V}\left(E\left(\Psi \mid \Gamma_{n}\right)\right), \quad \tilde{V}_{m}=\tilde{V}\left(E\left(\Psi \mid \Gamma_{m}\right)\right),
\end{gathered}
$$

where $\tilde{V}(\Psi)$ is the total variance of output $\Psi$ (i.e. $A_{y} / D, A_{x} / D$ or $\left.A_{m} / D\right), E(\Psi \mid \Gamma)$ is the conditionally expected value obtained by averaging all possible outcomes of $\Psi$ based on the (individual or multiple) $\Gamma$ input changes, and $\tilde{V}(E(\Psi \mid \Gamma))$ denotes the variance of expectations. Eqs. (20) and (21) can be assessed using the opensource GSAT toolbox in Matlab (Cannavó, 2012).

Note that attention should be paid to the coexisting upper/lower branches exhibiting a jump for the cylinder with low $m^{*}$ when evaluating PC, SRC, $\mathrm{R}^{2}$ and SI for sensitivity analyses. To avoid the mixed statistics, upper- and lower-branch responses in this range are separated prior to the post-processing. Although MCS create a high number of data through several runs, the total required computational effort and data storage are indeed relatively minimal based on the proposed reduced-order model.

\subsection{Sensitivity to Reynolds Number}

The hydrodynamic characteristics of flows around circular cylinders are governed by several factors including Re, the turbulence intensity of incoming flows and the cylinder surface roughness. In addition, the measured hydrodynamic forces of vortex shedding formations are influenced by experimental arrangement parameters such as the cylinder aspect ratio, wall proximity, free surface, blockage and three-dimensionality (Blevins, 1990; Sumer and Fredsoe, 2006; Zdravkovich, 1997). With these variable conditions in different testing facilities, the collected hydrodynamic data are often scattered, leading to some uncertainty contributions to the model calibration and prediction comparison. In this study, experimental data of smooth cylinders in steady flows with free or very low turbulence are considered regardless of their experimental arrangements.

As Re affects the flow regime and vortex shedding pattern, this second part aims to incorporate the Re dependence into the prediction model for investigating the Re sensitivity of 2-DOF VIV outcomes (Section 4.3). This is accomplished by considering (i) the Re-dependent hydrodynamic coefficients $\left(\mathrm{St}, C_{D 0}, C_{L 0}, \bar{C}_{D 0}\right)$ which affect several dimensionless quantities (Table 1), and (ii) the Re-dependent amplitude function $\left(A^{*}\right)$ which, in turn, influences the mean drag $\left(\bar{C}_{D}\right)$ through Eq. (13). Re is varied with $V$ through $\mathrm{Re}=V D / v$ where $v$ is the fluid kinematic viscosity. To account for the sole Re variation, $V_{r}$ is assigned and fixed. This implies that $D$ and/or $f_{n}$ is adjusted through $V_{r}=V / f_{n} D$, as carried out in Blevins and Coughran (2009) with varying $D$ and Re. In regard to the flow regime, attention is placed on the subcritical flows with the approximate range of $1 \times 10^{3}<\operatorname{Re}<3 \times 10^{5}$ (Sumer and Fredsoe, 2006). A critical flow regime $\left(3 \times 10^{5}<\operatorname{Re}<3.5 \times 10^{5}\right)$ is disregarded as the present wake-body oscillators presently exclude the possible occurrence of a non-zero mean lift force in this Re range (Schewe, 1983). The supercritical flow regime at higher Re is also disregarded due to the lack of empirical functions and experimental VIV data.

To account for the Re dependence of St, empirical functions based on a compilation of experimental data in Norberg (2003) are considered and plotted in Fig. 3a. These functions read

$$
\mathrm{St} \approx 0.2139-4 / \mathrm{Re} \quad ; 325 \leq \mathrm{Re} \leq 1.6 \times 10^{3},
$$




$$
\begin{array}{ll}
\mathrm{St} \approx 0.1853+0.0261 \exp \left(-0.9\left[\log \left(\operatorname{Re} / 1.6 \times 10^{3}\right)\right]^{2.3}\right) & ; 1.6 \times 10^{3} \leq \mathrm{Re} \leq 1.5 \times 10^{5}, \\
\mathrm{St} \approx 0.1848+0.00086\left[\operatorname{Re} / 1.5 \times 10^{5}\right]^{4.6} & ; 1.5 \times 10^{5} \leq \mathrm{Re} \leq 3.4 \times 10^{5} .
\end{array}
$$

Norberg (2003) also proposed the Re-based empirical functions for the sectional root-mean-squared lift coefficients of stationary cylinders. These statistical functions which account for the lift force fluctuations are herein considered for $C_{L 0}$ as follows. The $C_{L 0}$ variation and sample data are plotted in Fig. $3 \mathrm{~b}$.

$$
\begin{array}{ll}
C_{L 0} \approx 0.045+1.05\left[1-\operatorname{Re} / 1.6 \times 10^{3}\right]^{4.5} & ; 260 \leq \operatorname{Re} \leq 1.6 \times 10^{3}, \\
C_{L 0} \approx 0.045+3\left[\log \left(\operatorname{Re} / 1.6 \times 10^{3}\right)\right]^{4.6} & ; 1.6 \times 10^{3} \leq \mathrm{Re} \leq 5.4 \times 10^{3}, \\
C_{L 0} \approx 0.52-0.06\left[\log \left(\operatorname{Re} / 1.6 \times 10^{3}\right)\right]^{-2.6} & ; 5.4 \times 10^{3} \leq \mathrm{Re} \leq 2.2 \times 10^{5}, \\
C_{L 0} \approx 0.09+0.43 \exp \left[-10^{5}\left(\operatorname{Re} / 10^{6}\right)^{10}\right] & ; 2.2 \times 10^{5} \leq \mathrm{Re} \leq 3.4 \times 10^{5} .
\end{array}
$$

Experimental $C_{D 0}$ data are scarce and King (1977) suggested that $C_{D 0} \approx 0.2$ may be used for all $\operatorname{Re}$ as applied in Currie and Turnbull (1987). Nevertheless, Bishop and Hassan (1964a) and Cheung and Melbourne (1983) reported very few $C_{D 0}$ data as plotted in Fig. $3 \mathrm{c}$. In the lower Re $\leq 5.8 \times 10^{4}$ range, the averaged $C_{D 0} \approx$ 0.06 is herein assumed whereas a new curve-fitting $C_{D 0}$ function is introduced for a higher Re range as

$$
C_{D 0} \approx-0.4408 \log \left(\operatorname{Re} / 10^{5}\right)+0.2478 \quad ; 5.8 \times 10^{4} \leq \mathrm{Re} \leq 3.0 \times 10^{5} .
$$

As for $\bar{C}_{D 0}$, experimental data compiled by Schlichting and Gersten (2017) are considered and plotted in Fig. 3d. Accordingly, new empirical $\bar{C}_{D 0}$ curve-fitting functions are introduced as follows:

$$
\begin{array}{ll}
\bar{C}_{D 0} \approx 1.095\left(\mathrm{Re} / 10^{3}\right)^{-0.179} & ; 1.0 \times 10^{3} \leq \mathrm{Re} \leq 2.1 \times 10^{3}, \\
\bar{C}_{D 0} \approx 1.164\left(\mathrm{Re} / 10^{3}\right)^{0.033}-0.493\left(10^{3} / \mathrm{Re}\right) & ; 2.1 \times 10^{3} \leq \mathrm{Re} \leq 1.0 \times 10^{5}, \\
\bar{C}_{D 0} \approx 0.019\left(\mathrm{Re} / 10^{5}\right)^{3}-0.173\left(\mathrm{Re} / 10^{5}\right)^{2}+0.323\left(\mathrm{Re} / 10^{5}\right)+1.182 & ; 1.0 \times 10^{5} \leq \mathrm{Re} \leq 3.0 \times 10^{5} .
\end{array}
$$

Figure 3 shows the variation ranges of $0.18<\mathrm{St}<0.22,0.05<C_{L 0}<0.55,0.06<C_{D 0}<0.35$ and $0.95<\bar{C}_{D 0}<1.35$ which are more versatile than the usually fixed $\mathrm{St}=0.2, C_{L 0}=0.3, C_{D 0}=0.2$ and $\bar{C}_{D 0}=1.2$ in the wake oscillators. Eqs. (30)-(32) may be combined with Eq. (13), dependent on $A^{*}$ and $\bar{k}$, for approximating the dynamically amplified steady $\operatorname{drag}\left(\bar{C}_{D}\right)$ as Re is varied. To capture the $A^{*}$ variation as a function of Re as well as $m^{*} \xi$, the following correlation for a maximum response may be used (Govardhan and Williamson, 2006),

$$
A^{*}=\left(1-1.12 \alpha+0.3 \alpha^{2}\right) \log \left(0.41 \mathrm{Re}^{0.36}\right),
$$

where $\alpha=\left(m^{*}+C_{a}\right) \xi$ and $C_{a}=1$. Note that Eq. (33) has been validated to be satisfactory for $500<\operatorname{Re}<3.3 \times 10^{4}$ and this range may be further valid at a higher subcritical Re as noted by Govardhan and Williamson (2006). As Eq. (33) has been derived based on 1-DOF VIV tests, it is most suitable for 2-DOF VIV analysis for cylinders with high $m^{*}>6$ where the effects of in-line VIV and stiffness nonlinearities are negligible due to the lowering cross-flow response. In the absence of amplitude correlation from 2-DOF VIV tests, Eq. (33) is herein utilised for all $m^{*}$ by assuming that the drag magnification is proportional to a predominant $y$ response as experimentally observed in Stappenbelt et al. (2007). The effects of in-line VIV and geometric nonlinearities can be accounted for by tuning $\bar{k}$ instead as discussed in Section 4.3. 
To capture the Re dependence of the lift force wake oscillator, Eq. (35) from Facchinetti et al. (2004) and Eq. (33) may be combined to arrive at a unique relationship for the two wake coefficients $\Lambda_{y}$ and $\varepsilon_{y}$ as

$$
\frac{\Lambda_{y}}{\varepsilon_{y}}=\left[\frac{4 \mathrm{~S}_{\mathrm{G}}+16 \pi^{2} \mathrm{St}^{2} \gamma}{C_{L 0}}\right]\left[\left(\left(\frac{2 \mathrm{~S}_{\mathrm{G}}+8 \pi^{2} \mathrm{St}^{2} \gamma}{C_{L 0}}\right)\left(1-1.12 \alpha+0.3 \alpha^{2}\right) \log \left(0.41 \mathrm{Re}^{0.36}\right)\right)^{2}-1\right],
$$

where $\mathrm{S}_{\mathrm{G}}=8 \pi^{2} \mathrm{St}^{2} \mu \xi=2 \pi^{3} \mathrm{St}^{2} \alpha$ is the so-called Skop-Griffin parameter (Skop and Balasubramanian, 1997). It is worth remarking that Eq. (34) depends on both physical parameters $\left(m^{*}, \xi\right)$ and Re-dependent hydrodynamic properties (St, $\left.C_{L 0}, \gamma\left(\bar{C}_{D}, \mathrm{St}\right)\right)$ deduced from Eqs. (22)-(32). The empirical $\varepsilon_{y}$ expression depending on $m^{*}$ may be specified through Eq. (14) so that a sole variation in $\Lambda_{y}$ is achieved. The $\Lambda_{y} / \mathcal{E}_{y}$ plots as function of Re and $m^{*}$ will be presented in Section 4.3 together with the analysis of model sensitivity to Re.

\section{Parametric Investigations}

Insights into the model empirical sensitivities, output uncertainties and effects of fluid-structure parameters are now highlighted. Convergence tests have been performed with increasing $N$ in MCS such that 1000 series of random input vectors $\Upsilon(N=1000)$ provide converging outputs with invariant means and standard deviations. Table 2 summarizes the assumed mean values of input variables used in MCS. These values have been deduced by Srinil and Zanganeh (2012) through calibration with several 2-DOF VIV experimental results (Blevins and Coughran, 2009; Jauvtis and Williamson, 2004; Stappenbelt et al., 2007). By considering the cylinders with a fixed $\xi=0.6 \%$ and varied $m^{*}=3.68,5.19$ and 8.76 (Stappenbelt et al., 2007), cross-flow amplitudes $\left(A_{y} / D\right)$, in-line amplitudes $\left(A_{x} / D\right)$ and in-line mean displacements $\left(A_{m} / D\right)$ are evaluated in the case of varying $V_{r}$ to capture initial, upper or super-upper (where maximum amplitude occurs), and lower branch responses (Jauvtis and Williamson, 2004). In the following, the output components are explained by also demonstrating the capability of the present wake-cylinder oscillators in capturing basic VIV features.

\subsection{Response Output Variations}

The Re-independent model is first considered to demonstrate output components. With $m^{*}=3.68, V_{r}=9$ and initial conditions $x=y=\dot{x}=\dot{y}=\dot{p}=\dot{q}=0, p=2$ and $q=2$, Fig. 4 displays the response time histories including transient dynamics of $x$ (4a), $p$ (4b), $y(4 \mathrm{c})$ and $q$ (4d) associated with Eqs.(5)-(8). Overall, the steady-state responses are achieved when $t>150$, exhibiting a perfect periodicity and single harmonic oscillation frequency. Maximum, mean and minimum $x$ components can be extracted from Fig. 4 a, establishing $A_{x} / D$ and $A_{m} / D$. A zero mean in $y$ response is guaranteed in Fig. $4 \mathrm{c}$ with $A_{y} / D$ being equal to the maximum (or the absolute minimum) $y$ component. The time histories of wake variables $p$ (Fig. 4b) and $q$ (Fig. 4d) reveal the amplified magnitudes ( $p \approx 3.5, q \approx 21.6$ ) which are notably greater than their initial conditions ( $p=q=2$ ) owing to the VIV fluid-structure interaction effect. A zero mean in $p$ and $q$ responses justifies a realistic simulation of fluctuating drag and lift force components through the van der Pol equations forced by the cylinder accelerations.

With the same $m^{*}=3.68$, output diagrams of $A_{y} / D, A_{x} / D$ and $A_{m} / D$ with varying $V_{r}$ are presented in Figs. $5 \mathrm{a}, 5 \mathrm{~b}$ and $5 \mathrm{c}$, respectively. For each $V_{r}, 100 \mathrm{MCS}$ are exemplified, and the initiated displacement and velocity conditions of all $x, y, p$ and $q$ are fixed; otherwise they will further affect the free-oscillation variability. Several key observations can be made from Fig. 5. Pure in-line VIV responses (Currie and Turnbull, 1987; King, 1977) 
are captured in the range of $2<V_{r}<3$ whose very small amplitudes are insensitive to the input randomness (Fig. $5 \mathrm{~b})$. With regard to the main 2-DOF VIV range, a greater output certainty appears in an initial $\left(V_{r}<4\right)$ and lower ( $V_{r}>8$ ) branch range with a high repeatability of $A_{y} / D<0.5$ (Fig. 5a) and $A_{x} / D<0.05$ (Fig. 5b). In these ranges, $A_{m} / D$ values (Fig. 5 c) increase linearly with $V_{r}$ due to the increasing steady drag force proportional to $V^{2}$ (Eq.3). Within the middle upper branch range $4<V_{r}<14, A_{m} / D$ are substantially enlarged due to the dynamic drag amplification coupled with $A_{y} / D$ and $A_{x} / D$. In this main resonant range, the multivariable output uncertainties increase visibly as amplitudes and mean displacements increase. The maximum responses are in the approximated ranges of $1<A_{y} / D<1.4,0.1<A_{x} / D<0.3$ and $0.25<A_{m} / D<1.5$, showing the input sensitivity and output variation when compared with experimental data (Stappenbelt et al., 2007) whose maximum $A_{y} / D \approx 1.4$ and $A_{x} / D \approx 0.23$ deduced by averaging the top $10 \%$ of the half peak-to-peak values from the experimental time histories. In Figs. 5a-5c, multiple locations of response jumps suggest a critical parametric range where upper and lower branches coexist $\left(8<V_{r}<14\right)$. This multiple solution range is susceptible to initial conditions which are uncertainly random in nature. Therefore, there is a wider possibility that the cylinder response may undergo and switch between greater or smaller $x-y$ responses resulting in the cylinder static drift variations.

With $V_{r}=9$, the $x-y$ orbital motions associated with the upper branch are displayed in Fig. $5 \mathrm{~d}$ (left-to-right flow direction) which exhibits various figure-of-eight trajectories associated with dual 2:1 resonances (Dahl et al., 2010; Zanganeh and Srinil, 2014). The relatively similar patterns with two lobs pointing downstream are remarked. By inspecting the motion paths, the majority of the eight figures follow the counter-clockwise (clockwise) direction at their top (bottom) parts as exemplified by arrows in Fig. 5d. These are typical upperbranch patterns experimentally observed (Blevins and Coughran, 2009; Dahl et al., 2010; Sanchis et al., 2008; Srinil et al., 2013). The $x-y$ phase differences are mostly about $315^{\circ}$ with $x$ leading $y$ (Jauvtis and Williamson, 2004). Such repetitive resembling features suggest the rather quantitative than qualitative effect of the input randomness. This remark is also supportive to both the amplitude (Figs. 5a and 5b) and mean (Fig. 5c) response diagrams where the self-limiting and jump characters are all maintained.

With 1000 MCS for each $V_{r}$, the means and standard deviations of $A_{y} / D, A_{x} / D$ and $A_{m} / D$ outputs associated with the upper branches are plotted in Fig. 6 for different $m^{*}=3.68,5.19$ and 8.76. Within the critical range (i.e. $V_{r}>8$ for $m^{*} 3.68,6<V_{r}<8$ for $m^{*}=5.19,6<V_{r}<7$ for $m^{*}=8.76$ ), it is necessary to separate the upperbranch MCS data from the lower-branch ones to avoid the mixed statistics which could be misleading. In so doing, a threshold $A_{y} / D$ value (i.e. 1.0 for $m^{*}=3.68,0.8$ for $m^{*=5.19}$, and 0.5 for $m^{*=8.76)}$ is introduced to group the 2-DOF VIV data. The number of MCS is also increased such that the associated $A_{y} / D, A_{x} / D$ and $A_{m} / D$ in both branches account for at least $N=1000$ fulfilling the convergence. It can be seen from Fig. 6 that mean values of $A_{y} / D$ (Fig. 6a), $A_{x} / D$ (Fig. 6c) and $A_{m} / D$ (Fig. 6e) increase as $V_{r}$ is increased, and they decrease as $m^{*}$ is increased. This is expected from a lock-in resonance and mass ratio effect perspective (Jauvtis and Williamson, 2004). Nevertheless, in the peak response range, the maximum standard deviation of $A_{y} / D$ in Fig. 6b is about $12 \%$ for $m^{*}=3.68,9 \%$ for $m^{*}=5.19$ and $8 \%$ for $m^{*}=8.76$ ), being greater than those associated with $A_{x} / D$ in Fig. $6 \mathrm{~d}$ (up to $3.5 \%$ for $m^{*}=3.68$ ) and $A_{m} / D$ in Fig. 6f (up to $7.5 \% m^{*}=3.68$ ). This demonstrates a greater output variance associated with the larger $y$ response. When comparing with experimental data, these 
means and standard deviations may be useful for establishing the upper/lower response bounds.

To identify which individual input variables play the most influential role in the output uncertainty and to understand the interactive changes in multiple variables, a systematic sensitivity analysis is next carried out by also accounting for the effects of $m^{*}$ (Section 4.2 and 4.3) and Re (Section 4.3).

\subsection{Contribution, Relative Importance and Coupling of Wake-Cylinder Variables}

Of practical interest is the prediction of maximum $A_{y} / D, A_{x} / D$ and $A_{m} / D$ associated with the upper branches (e.g. $4<V_{r}<14$ in Fig. 5). By focusing on the model sensitivity to random changes in the six wake-cylinder variables $\left(\alpha_{x}, \alpha_{y}, \beta_{x}, \beta_{y}, \varepsilon_{y}, \Lambda_{y}\right), C_{D 0}, C_{L 0}, \bar{C}_{D 0}$ and St are fixed as in Table 2. Recalling Eqs. (5) and (7) that $\alpha_{x}$ and $\alpha_{y}$ govern the one-directional cubic stiffness $\alpha_{x} x^{3}$ and $\alpha_{y} y^{3}$ terms, $\beta_{x}$ and $\beta_{y}$ govern the bi-directionally coupled cubic stiffness $\beta_{x} x y^{2}$ and $\beta_{y} y x^{2}$ terms, and $\varepsilon_{y}$ and $\Lambda_{y}$ govern the fluctuating lift force through $q$ (Eq. 8). Both $\varepsilon_{x}$ and $\Lambda_{x}$ which affect the fluctuating drag force are not treated as random variables as they produce a negligible sensitivity as in Section 2.3. The scatterplots are first qualitatively analysed through $1000 \mathrm{MCS}$.

Both low $\left(m^{*}=3.68, V_{r}=9\right)$ and high $\left(m^{*}=8.76, V_{r}=6\right)$ mass ratio systems are considered and compared. A series of output variations in (a) $A_{y} / D$, (b) $A_{x} / D$ and (c) $A_{m} / D$ vs. the percentage changes in $\alpha_{x}, \alpha_{y}, \beta_{x}, \beta_{y}, \varepsilon_{y}$ and $\Lambda_{y}$ are presented in Fig. 7 for $m^{*}=3.68$ and 8.76 cases. Because a linear monotonic relationship is generally observed in the scatter data, a least-squares linear fitting is also applied to describe the mapping trend likelihood. With $m^{*}=3.68$, it is seen that $A_{y} / D$ points (Fig. 7a) with $\alpha_{x}$ and $\beta_{x}$ variations appear scattered but over two almost horizontal fitting lines. This implies the independence of $A_{y} / D$ on $\alpha_{x}$ and $\beta_{x}$. In other words, the $A_{y} / D$ variability is likely due to deviations in other input variables. This is feasible as $\alpha_{x}$ and $\beta_{x}$ govern the $x$ motion (Eq. 5). The negligible sensitivity to $\alpha_{y}$ is also noticed although the small $\beta_{y}$ dependence is realised by a slightly inclined fitting line. This suggests a weak dependence of $y$ geometric nonlinearities. Remarkably, $A_{y} / D$ outputs are significantly affected by both $\varepsilon_{y}$ and $\Lambda_{y}$ variations revealing their highly-inclined fitting lines albeit with the opposite effects. Accordingly, $A_{y} / D$ are likely to decrease with increasing $\varepsilon_{y}$ or decreasing $\Lambda_{y}$.

The sensitivity state of $A_{x} / D$ (Fig. 7b) becomes rather different from that of $A_{y} / D$ (Fig. 7a). It can be seen that, together with $\varepsilon_{y}, \Lambda_{y}$ and $\beta_{y}$, both $\alpha_{y}$ and $\beta_{x}$ variations now play a role in the model sensitivity. Due to the $x-y$ geometric coupling and greater $y$ response, both $y^{2}$ and $y^{3}$ nonlinearities appear to govern the smaller $x$ response: the former amplifies $A_{x} / D$ as $\beta_{x}$ increases due to the softening (quadratic) effect of $y^{2}$ whereas the latter reduces $A_{x} / D$ as $\alpha_{y}$ increases due to the stiffening (cubic) effect of $y^{3}$. The $\alpha_{x}$ variation is still negligible because of a small contribution of $x^{3}$ term. Owing to the amplified drag force within the main lock-in range, both $\alpha_{x}$ and $\beta_{x}$ variations affect $A_{m} / D$ outputs (Fig. 7c) due to the enlarged drifted $x$ (e.g. Figs. $4 \mathrm{a}$ and $5 \mathrm{c}$ ). On the contrary, variations in the $y$ counterparts $\left(\alpha_{y}, \beta_{y}\right)$ are negligible, suggesting that only the quadratic $y^{2}$ effect influences $A_{m} / D$ through $\beta_{x}$. This $y^{2}$ dependence is consistent with what has been discussed in Zanganeh and Srinil (2014) through a harmonic balance analysis. Similar to $A_{y} / D$ and $A_{x} / D$ plots, both $\varepsilon_{y}$ and $\Lambda_{y}$ variations significantly affect the $A_{m} / D$ output uncertainty.

Overall results in the case of $m^{*}=3.68$ highlight that the most influential input variables are $\varepsilon_{y}$ and $\Lambda_{y}$ associated with the fluctuating lift force wake oscillator which is coupled with both cross-flow (Eq. 7) and in- 
line (Eq. 5) cylinder oscillators. With higher $m^{*}=8.76$, output variations in $A_{x} / D(<0.05)$ and $A_{m} / D(<0.2)$ are considerably reduced against the changes in $\alpha_{x}, \alpha_{y}, \beta_{x}$ and $\beta_{y}$. These suggest a greater certainty in smaller predicted responses and reduced sensitivity to geometric nonlinearities. Nevertheless, $A_{y} / D$ outputs are still affected by the two key $\varepsilon_{y}$ and $\Lambda_{y}$ in the same manner as in the lower $m^{*=3.68}$ case. Based on these observations, the wake oscillators may be more suitable for high $m^{*}$ systems for which the fluid wake tends to be governed by the structural characteristics whose parameters are more well-defined. Greater care must be taken in the modelling of lower $m^{*}$ system especially for offshore underwater systems.

To quantitatively analyze the high number of scatter maps, the Pearson's correlation coefficients (PC) are now evaluated (Eq. 15) to gather more information on the model sensitivity to each input. With $V_{r}=6$, Fig. 8 presents the PC bar plots $[-1,1]$ for different input $\left(\alpha_{x}, \alpha_{y}, \beta_{x}, \beta_{y}, \varepsilon_{y}, \Lambda_{y}\right)$ vs. output $\left(A_{y} / D, A_{x} / D, A_{m} / D\right)$ variables for $m^{*}=3.68$ (red bars), $m^{*}=5.19$ (green bars) and $m^{*}=8.76$ (blue bars). The negative (positive) PC indicate the opposite (same) input-output correlations which give rise to the decreasing (increasing) outputs with increasing inputs. It can be seen in Fig. 8 that the strongest correlations $(|\mathrm{PC}|>0.5)$ are associated with $\varepsilon_{y}$ (negative PC) and $\Lambda_{y}$ (positive PC) in all outputs and $m^{*}$ cases. These confirm the qualitative observations through scatterplots in Fig. 7. The geometric nonlinearities $\left(\alpha_{x}, \alpha_{y}, \beta_{x}, \beta_{y}\right)$ play a less influential role in $A_{x} / D$ because of the smaller oscillation components which become even more decreased as $m^{*}$ is increased. Nevertheless, $\alpha_{y}$, and $\beta_{y}\left(\alpha_{x}\right.$ and $\left.\beta_{x}\right)$ significantly affect $A_{y} / D\left(A_{m} / D\right)$ with the hybrid \pm PC depending on $m^{*}$.

Next, the relative importance of individual input variables with respect to each $X$ - $Y$ output uncertainty is investigated through the regression analysis. For cylinders with $m^{*}=3.68,5.19$ and 8.76 , both absolute SRC (Eq. 18) and $\mathrm{R}^{2}$ (Eq. 19) values associated with the upper branches are reported in Tables 3, 4 and 5 for $A_{y} / D$, $A_{x} / D$ and $A_{m} / D$, respectively. For each set of SRC and $\mathrm{R}^{2}$, values in the top, middle and bottom rows correspond to $m^{*}=3.68,5.19$ and 8.76, respectively, and the underlined ones correspond to the maximum SRC. It should be noted that SRC values may be used to compare and rank the input variable importance (Ma et al., 1993) whereas the percentile $\mathrm{R}^{2}$ values can be used to justify how much the regression models account for the overall output uncertainty (Saltelli et al., 2000). Such ranking is beneficial from a modelling perspective if one wishes to reduce a calibration task vs. experimental data by focusing on the tuning of most influential variables. It can be seen that $\Lambda_{y}$, which models the fluid-cylinder interaction effect through the cylinder acceleration, has the greatest impact (SRC) on $A_{y} / D$ (Table 3), $A_{x} / D$ (Table 4) and $A_{m} / D$ (Table 5). By combining the deduced ranking from all Tables 3-5 which show a high accountability of output uncertainty with $\mathrm{R}^{2}$ being mainly more than $90 \%$, the next dominant variables appear mostly to be $\varepsilon_{y}, \alpha_{y}, \beta_{x}, \beta_{y}$ and $\alpha_{x}$, consecutively. This justifies that the top-3 most sensitive variables $\left(\Lambda_{y}, \varepsilon_{y}, \alpha_{y}\right)$ for the present wake-cylinder oscillators are associated with the greater $y$ response. For understanding the relative importance, the $m^{*}=3.68$ case is discussed as an example. The importance of $\Lambda_{y}$ is higher than that of the secondary influential $\varepsilon_{y}$ by about $12.3 \%(0.969$ vs. 0.863$)$ for $A_{y} / D, 7.8 \%$ (0.966 vs. 0.896$)$ for $A_{x} / D$ and $9.2 \%$ (0.958 vs. 0.877$)$ for $A_{m} / D$, and higher than that of the tertiary influential $\alpha_{y}$ by about $113.9 \%(0.969$ vs. 0.453$)$ for $A_{y} / D, 100.8 \%(0.966$ vs. 0.481$)$ for $A_{x} / D$ and $227 \%(0.958$ vs. 0.293) for $A_{m} / D$.

The model sensitivity to the coupled input variations is now appreciated by evaluating the normalized second-order Sobol' sensitivity indices SI (Eq. 20) whose values vary between zero and one (Cannavó, 2012). 
A higher SI close to one indicates a higher variance and thus a greater importance of the interaction between each two input variables on the output variance. With $V_{r}=6$, Fig. 9 illustrates a variety of high, medium and low SI levels for each multiple input interaction on the variance of $A_{y} / D, A_{x} / D$ and $A_{m} / D$ for different $m^{*}=$ 3.68 (Fig. 9a), 5.19 (Fig. 9b) and 8.76 (Fig. 9c). It is seen that, for all $m^{*}$ and output variables, the most important coupling effect is associated with $\Lambda_{y}$ and $\varepsilon_{y}$ which have been ranked by individual SRC (Tables 3-5) as the two most influential variables. This underlines the empirical coupling effect on the wake oscillator model, and how the wake coefficients are further cross-coupled with cylinder stiffness variables $\left(\alpha_{x}, \alpha_{y}, \beta_{x}, \beta_{y}\right)$ although the latter exhibit themselves relatively weaker geometrically-nonlinear coupling effects (i.e. mostly yellow $\mathrm{SI}<0.5$ ). As $m^{*}$ is increased, several variable coupling effects noticeably decrease although Fig. 9c reveals that the most-influential variable, interacting with all other variables, are still $\Lambda_{y}$ and $\varepsilon_{y}$. Overall coherent results suggest qualitatively and quantitatively that the lift force wake oscillator plays the most significant role in simulating cross-flow/in-line VIV. This enables us to effectively consider the derived Eq. (34) for the $\Lambda_{y}-\varepsilon_{y}$ variation as a function of Re as carried out in the following case studies.

\subsection{Influence of Reynolds Number on Maximum Responses}

As Re depends on $V, D$ and $v$, in this study Re is randomly varied due to a random change in $V$. This is sensible as the sea current practically changes due to the random environment. A model sensitivity to Re in predicting the maximum 2-DOF responses is now investigated by considering the variation of hydrodynamic coefficients (St, $C_{D 0}, C_{L 0}, \bar{C}_{D 0}$ ) as functions of Re (Fig. 3) as described in Section 3.2. The coupling of the two most influential variables $\Lambda_{y}$ and $\varepsilon_{y}$ identified in Section 4.2 is also treated as a function of Re through Eq. (34). To examine $\bar{C}_{D}$ variation, Eq. (13) is considered with $A^{*}$ function in Eq. (33) whereas $\bar{k}$ is obtained based on calibration of the maximum $\bar{C}_{D}$ with data of Stappenbelt et al. (2007) who suggested a lower $\bar{k}$ for a 2-DOF VIV than that (e.g. $\bar{k}=2.1$ ) for a 1-DOF VIV (Blevins, 1990). In this 2-DOF VIV study, the optimal $\bar{k}$ has been found to be about $0.85,0.60$ and 0.45 for $m^{*}=3.68,5.19$ and 8.76 , respectively.

Accordingly, the associated $\bar{C}_{D}$ and $\gamma=\bar{C}_{D} / 4 \pi$ St variations are plotted vs. Re in Figs. 10a and 10b which reveal their optimal peak values in a similar Re range $\left(\approx 1.35 \times 10^{5}-1.5 \times 10^{5}\right)$ for all $\mathrm{m}^{*}$, despite a slight variation in St (Fig. 3a). As $m^{*}$ is increased, $\bar{C}_{D}$ decreases owing to the decreased $A^{*}$, diminishing the stall parameter $\gamma$ (Fig. 10b) and, thus, the damping effect (Eq. 11). A range of maximum $\bar{C}_{D}$ of about 2.18-2.98 for $8.76<m^{*}<3.68$ is quantitatively similar to that of about $1.98-2.80$ for $10.63<m^{*}<2.36$ in Stappenbelt et al. (2007). Both $\bar{C}_{D}$ and $\gamma$ plots in Figs. 10a and 10b enable the wake oscillators to achieve a greater flexibility in capturing the influence of $\operatorname{Re}$ as well as $m^{*}$, in contrast to the model with a typically fixed $\bar{C}_{D}=2$ and $\gamma=0.8(\mathrm{St}=0.2)$ (Facchinetti et al., 2004; Srinil and Zanganeh, 2012). From Fig. 10b, the maximum $\gamma$ increases by about 36.5\% as $m^{*}$ is decreased by about $58 \%$ from 8.76 to 3.68 . This will affect the 2-DOF response prediction through Eq. (34) depending also on other system parameters.

The plots of $\Lambda_{y} / \varepsilon_{y}$ vs. Re based on the variable $\gamma$ and fixed $\gamma=0.8$ models are compared in Fig. 10c for $m^{*}$ $=3.68$ and Fig. $10 \mathrm{~d}$ for $m^{*}=8.76$. Both qualitative and quantitative differences can be noticed. For a given 
$\operatorname{Re}, \Lambda_{y} / \mathcal{E}_{y}$ of the variable $\gamma$ model decreases as $m *$ is increased whilst $\Lambda_{y} / \mathcal{E}_{y}$ of the fixed $\gamma$ model slightly increases as $m^{*}$ is increased. The latter model also provides overestimated $\Lambda_{y} / \varepsilon_{y}$ values. By considering the more versatile, variable $\gamma$ model and keeping $\varepsilon_{y}$ fixed according to the $m^{*}$-based function (Eq. 14), the plots of $\Lambda_{y}$ vs. Re are shown in Fig. 10e. Alternatively, by considering the fixed $\Lambda_{y}=12$ (Table 2), the plots of $\varepsilon_{y}$ vs. Re are shown in Fig. 10f. It can be seen that, as Re is increased, $\Lambda_{y}$ increases whereas $\varepsilon_{y}$ decreases, with the latter converging to a certain value $\left(\approx 0.007\right.$ for $m^{*}=3.68,0.008$ for $m^{*}=5.19,0.015$ for $\left.m^{*}=8.76\right)$ at a higher Re. The parametric variations in both Figs. 10e and 10f suggest how the fluid-structure interaction effect could be enhanced as the coupling $\left(\Lambda_{y}\right)$ or wake damping $\left(\varepsilon_{y}\right)$ becomes greater or smaller with increasing Re, respectively. This feature supports some experimental cross-flow VIV studies reporting the increasing Re effect (Blevins and Coughran, 2009; Govardhan and Williamson, 2006).

To demonstrate the Re influence on the 2-DOF VIV, experimental model of Stappenbelt et al. (2007) is considered. Their tests reported the Re range of about $3.7 \times 10^{4}-4.8 \times 10^{4}$ associated with the maximum responses at $5<V_{r}<9$ for $12.96<m^{*}<2.36$. By following the recommended practice DNV-RP-F204 (2010) which suggests using the lognormal Gaussian distribution for environmental variables with a coefficient of variation $\mathrm{CoV}$ (the standard deviation divided by the mean) in the range of 0.05-0.1, we apply this lognormal distribution for Re (i.e. $V$ ) with $\mathrm{CoV}=0.08$ as in Low and Srinil (2016), and a mean value of Re associated with $V$ producing the peak response reported in Stappenbelt et al. (2007). For the representative cases of $m^{*}=3.68$ and 5.19, the mean Re is about $3.69 \times 10^{4}$ and $3.36 \times 10^{4}$, respectively. With this assumed distribution, a random sampling is performed to generate 1000 Re samples whose all values are found to be positive for MCS. Then, for each random Re, the associated St (Fig. 3a), $C L_{0}$ (Fig. 3b), $C D_{0}$ (Fig. 3c), $\bar{C}_{D}$ (Fig. 10a), $\gamma\left(\right.$ Fig. 10b) and $\Lambda_{y}$ (Eq. 34 and Fig. 10e) are re-evaluated, depending on the specified $m^{*}$ and associated $\varepsilon_{y}$. All 1000 Re-dependent $\Lambda_{y} / \varepsilon_{y}$ values are applied to MCS with other fixed variables $\left(\varepsilon_{x}, \Lambda_{x}, \alpha_{x}, \beta_{x}, \alpha_{y}, \beta_{y}\right)$ as in Table 2 to maintain the Re effect through only $\Lambda_{y}$. By specifying $V_{r}=6,6.5$ and 7, Fig. 11 exemplifies the plots of maximum $A_{y} / D, A_{x} / D$ and $A_{m} / D$ vs. Re variation for $m^{*}=3.68$ (Fig. 11a) and $m^{*}=5.19$ (Fig. 11b). Note that, in the higher $m^{*}$ case

(Fig. 11b), the outputs at $V_{r}=7$ are associated with a response jump (Stappenbelt et al., 2007), and this is partially captured at lower Re. For the present $V_{r}$ range, $A_{y} / D$ outputs are relatively independent of Re variation. This feature occurring in the 2-DOF VIV has been discussed by Jauvtis and Williamson (2004) who reported a narrow range of maximum $A_{y} / D(\approx 1.36-1.51)$ for $3.3 \times 10^{3}<\operatorname{Re}<1.53 \times 10^{4}$ and $m^{*}<5(0.0017<\alpha<0.0255)$. Nevertheless, the influence of $\operatorname{Re}$ on $A_{x} / D$ and $A_{m} / D$ is more noticeable as these outputs become greater as $\operatorname{Re}$ or $V_{r}$ is increased prior to a response jump.

\subsection{Validation of Assumptions by Statistical Hypothesis Testing}

Numerical MCS in Section 4.3 rely on several assumptions and hypotheses whose empirical functions have been deduced from or calibrated with some experimental data in relevant literature. These entail the following model implementations influencing the response predictions:

- Use of empirical functions Eq. (13) for $\bar{C}_{D}$, Eq. (14) for $\varepsilon_{y}$ and Eq. (33) for $A^{*}$,

- Use of lognormal Gaussian distribution of Re through the variable $V$ (DNV, 2010),

- Use of coefficient of variation $(\mathrm{CoV})$ of 0.08 for Re in all $m^{*}$ cases, 
- Use of a reference mean Re value associated with the experimentally-observed peak response,

- Variations of Re-dependent St, $C_{L 0}, C_{D 0}$ and $\bar{C}_{D 0}$ through Eqs. (22)-(32),

- Use of empirical relationship of the most influential variables $\varepsilon_{y}$ and $\Lambda_{y}$ through Eq. (34),

- Use of other fixed coefficients $\varepsilon_{x}, \Lambda_{x}, \alpha_{x}, \beta_{x}, \alpha_{y}$ and $\beta_{y}$ as in Table 2,

- Synchronization range of $4<V_{r}<9$ in which a two-dimensional maximum response occurs.

It is worth justifying whether/when the predicted maximum response based on the above considerations is statistically acceptable. This may be achieved by performing a standard hypothesis testing with the analysis of probability or $p$-values through the so-called $t$-test (Krzywinski and Altman, 2013). Herein, the $p$-value is defined as the probability of getting a predicted maximum response equal to or more extreme than what was experimentally observed, under the null hypothesis in which the population mean is assumed to be equal to the hypothesized mean (i.e. the experimental peak response). The analysis steps are briefly described as follows.

a) Based on experimental data of Stappenbelt et al. (2007), a reference Re where the maximum response occurs is identified and used as the hypothesized mean for its probability distribution, for each $m^{*}$.

b) With this mean Re and specified $\mathrm{CoV}$, a random sampling is performed to generate 1000 Re values.

c) Our aim is to determine the optimal $\bar{k}$ for use with Eq. (13). For a given $\bar{k}$ and variable Re, a series of random inputs are generated through the above-implemented functions.

d) MCS are then performed for each $V_{r}$ to predict the maximum response outputs whose mean value can be evaluated. $V_{r}$ is then varied in the specified lock-in $\left(4<V_{r}<9\right)$ range with an increment of 0.5 . Hence, several sample mean values are obtained.

e) The one-sample $t$-test is performed based on the averaging of sample means and the pre-defined confidence interval to check whether the null hypothesis is acceptable. This occurs when the obtained $p$-value (i.e. the summed two-tailed areas in the $t$-distribution diagram of obtained mean values) is greater than the confidence level (so-called alpha percentile) of 0.05 based on the $95 \%$ confidence interval (Melchers, 1999). If acceptable, there is no statistical significance between the averaged mean (prediction) and the hypothesized mean (experimental data).

f) To achieve the optimal solution for each $m^{*}, \bar{k}$ is varied with an increment of 0.1 within the range of 0.1-1, and the above b)-e) steps are repeated.

Results of $p$-values against $\bar{k}$ variation for $m^{*}=3.68,5.19$ and 8.76 are plotted in Fig. 12 together with the associated curve-fitting trends. It can be realized that MCS results are statistically significant when considering the lowest $m^{*}=3.68$ whose $p$-values are all less than 0.05 . This is as expected since the above functions Eqs. (13), (33) and (34) were proposed based on 1-DOF cross-flow VIV experiments. The in-line VIV and mean displacements should also play a great role in the 2-DOF VIV prediction for such a low $m^{*}$. In higher $m^{*}=5.19$ and 8.76 cases where the in-line response effect becomes less influential, $p$-values are mostly greater than 0.05 , entailing the optimal $\bar{k} \approx 0.6$ and 0.45 , respectively. Hence, the most confident prediction is in the highest $m^{*}$ case. Such $\bar{k}$ values were applied in Fig. 11. Overall, the present assumptions and hypotheses are justified for the intermediate to higher $m^{*}(\geq 5)$ range in which the aforesaid empirical coefficients, functions and optimal $\bar{k}$ values may be applied to a 2-DOF VIV prediction. 


\subsection{Effect of Correlation between Random Variables}

It is worthwhile to appreciate the effect of correlation between random variables influencing the coupled crossflow/in-line VIV prediction. By way of examples, the two empirical variables $\bar{C}_{D}$ (Eq. 13) and $A^{*}$ (Eq. 33) are considered to be interdependently correlated. This correlation is reasonable since, under the same flow velocity and wake, the maximum response $A^{*}$ in cross-flow direction is subject to the lift force which should be coupled with the amplified mean drag force $\bar{C}_{D}$ depending on $A^{*}$. Based on the limited experimental data of $A^{*}$ vs. $\bar{C}_{D}$ gathered from Stappenbelt et al. (2007) and Stappenbelt and O'Neill (2007) for eight $m^{*}(2.36,3.01,4.89$, $6.54,6.75,8.49,8.76,10.63)$ cases, the associated correlation coefficient $\left(\rho_{\times 1 \times 2}\right)$ of $A^{*}$ and $\bar{C}_{D}$ is evaluated which is found to be equal to about 0.779 . This suggests a high degree of their interdependence.

In this study, the Nataf Transformation Method (NTM), which is applicable to a normal or lognormal distribution with a positive correlation, is applied to construct the joint probability density function (jpdf) of the correlated random variables (Saltelli et al., 2000). By letting the variation of $\bar{C}_{D}$ as $X_{1}$ and that of $A^{*}$ as $X_{2}$ from its experimental mean value, these two original dependent variables are first transformed to the correlated Gaussian variables $Y_{1}$ and $Y_{2}$, according to $Y_{\mathrm{i}}=\Phi^{-1}\left[F_{\mathrm{xi}}\left(X_{\mathrm{i}}\right)\right]$ for $\mathrm{i}=1,2$, where $\Phi^{-1}[$.$] is the inverse standard$ normal cumulative distribution function and $F_{\mathrm{Xi}}($.$) is the marginal cumulative distribution function (cdf). The$ NTM approximates the jpdf of $X_{1}$ and $X_{2}\left(f_{\mathrm{X} 1 \times 2}\right)$ through

$$
f_{x 1 X 2}\left(X_{1}, X_{2}\right)=f_{X 1}\left(X_{1}\right) f_{X 2}\left(X_{2}\right) \frac{\phi_{2}\left(Y_{1}, Y_{2}, \rho_{y 1 y 2}\right)}{\phi\left(Y_{1}\right) \phi\left(Y_{2}\right)},
$$

where $f_{\mathrm{Xi}}($.$) is the marginal pdf, \phi_{1}($.$) and \phi_{2}($.$) are, respectively, the univariate and bivariate standard normal$ pdf, and $\rho_{\mathrm{Y} 1 \mathrm{Y} 2}$ is the correlation coefficient for $Y_{1}$ and $Y_{2}$. In a bivariate case, one may assume $\rho_{\mathrm{Y} 1 \mathrm{Y} 2} \approx \rho_{\mathrm{X} 1 \mathrm{X} 2}$, with an error $<1 \%$ (Melchers, 1999). The next step is to transform the correlated standard normal variables $Y_{\mathrm{i}}$ to the uncorrelated ones $U_{\mathrm{i}}$, and this may be performed using the Cholesky factorization as in Low and Srinil (2016). Accordingly, the transformation in the bivariate case may be written as $Y_{1}=\sqrt{1-\rho_{Y 1 Y 2}^{2}} U_{1}+\rho_{Y 1 Y 2} U_{2}$ and $Y_{2}=U_{2}$. Hence, the transformation between the physical and standard normal space reads

$$
X_{1}=F_{X 1}^{-1}\left[\Phi\left(\sqrt{1-\rho_{Y 1 Y 2}^{2}} U_{1}+\rho_{Y 1 Y 2} U_{2}\right)\right], \quad X_{2}=F_{X 2}^{-1}\left[\Phi\left(U_{2}\right)\right] .
$$

As the original dependent variables are expressed in terms of the independent standard normal variables, MCS can be carried out with 1000 random Re values (as in Sections 4.3 and 4.4) and the correlation of $\bar{C}_{D}$ and $A^{*}$ based on Eq. (36). With $1<V_{r}<15$ range and a given $m^{*}=3.68$, numerical results of cross-flow and inline amplitudes in the correlated case normalized by those in the uncorrelated case $\left(A_{R}\right)$ are illustrated vs. the coexisting $U_{1}[-0.5,0.5]$ and $U_{2}[-1.5,2]$ in Fig. 13, with a positive (negative) $U_{\mathrm{i}}$ indicating the randomly increased (decreased) variation of $\bar{C}_{D}\left(U_{1}\right)$ and $A^{*}\left(U_{2}\right)$ from its experimental mean value of about $2.45\left(U_{1}=0\right)$ and $0.94\left(U_{2}=0.25\right)$, respectively. Note that the reported $A_{R}$ values are based on the averaged results of all considered $V_{r}$. It is noticed in Fig. 13 that both correlated $\left(\rho_{\mathrm{y} 1 \mathrm{y} 2} \approx 0.779\right)$ and uncorrelated $\left(\rho_{\mathrm{Y} 1 \mathrm{Y} 2}=0\right)$ cases yield comparable predictions as $U_{1}>0$ and $U_{2}>0.25$ : this implies that the experimentally referenced mean establishes a lower bound for the correlation effect to be negligible $\left(A_{R}=1\right)$. On the contrary, a high fluctuation 
of cross-flow amplitude ratios (Fig. 13a) occurs when $U_{1}<0$ and $U_{2}<0.25$, which suggests a greater correlation effect area in which the maximum response prediction could be underestimated $\left(A_{R}>1\right)$ or overestimated $\left(A_{R}<1\right)$ by the uncorrected variable model. Nevertheless, this correlation effect becomes reduced as $A_{R}$ gets closer to 1 for the associated in-line VIV with smaller amplitudes (Fig. 13b). In accordance with the analyses in Sections 4.2-4.4, the effect of interdependent variables appears to be also amplitude-dependent.

Such an insight into the correlation effect of different empirical variables should be further explored by accounting for more experimental datasets. This is a challenging task since experimental 2-DOF VIV studies in the literature are subject to different setups, flow-cylinder properties and measurement/post-processing uncertainties, complicating the model specification requiring the fixed vs. variable inputs which are also related to physical parameters. New modelling-inspired experiments with controlled parameters (e.g. by keeping $V_{r}$ fixed while varying Re, and vice versa) should be carried out within a unique testing framework.

\section{Discussion}

Quantitative predictions of coupled cross-flow/in-line VIV and in-line mean displacements in Section 4 have proved to be challenging due to the multivariate interactions depending on several fluid-structure parameters. Undoubtedly, a universal set of empirical coefficients capturing all possible response scenarios does not exit. Notwithstanding this, the present study has shown the feasibility of improving the use of wake-cylinder oscillators through a series of sensitivity analyses enabling the identification of the most influential variables affecting the output uncertainties. To further improve the model prediction capability and comparison with experimental results, some other key aspects are remarked as follows.

(i) The cylinder acceleration coupling term in Eqs. (6) and (8) has been implemented for a 2-DOF VIV model, following the recommendation of Facchinetti et al. (2004) who studied a 1-DOF cross-flow-only VIV. It would be worthwhile to explore whether and how the velocity coupling (Ma and Srinil, 2017; Skop and Balasubramanian, 1997) affects the bi-dimensional fluid-cylinder dynamics sensitivity, and whether overall $x$ $y$ response predictions could be quantitatively improved. This is because the 2-DOF vs. 1-DOF VIV behaviours are relatively distinctive for cylinders with a low mass ratio $m^{*}<6$ (Jauvtis and Williamson, 2004).

(ii) Cross-flow $\left(y^{3}, y x^{2}\right)$ and in-line $\left(x^{3}, x y^{2}\right)$ geometric nonlinearities more govern $A_{x} / D$ and $A_{m} / D$ than $A_{y} / D$ through $\alpha_{y}, \beta_{y}, \alpha_{x}$ and $\beta_{y}$ (Eqs. 5 and 7) due to the $x-y$ coupling and amplified drift-induced stretching effects. With $m^{*}>6$, variations in these variables play a negligible role in all the resulting outputs, and, therefore, the associated sensitivity investigation could be skipped to minimize time during parametric studies.

(iii) Correlation between the most influential variables $\left(\Lambda_{y}, \varepsilon_{y}\right)$ governing the lift wake oscillator and physical parameters Re and $m^{*}$ has been presented in Section 3.2 with the case studies in Section 4.3 based on a specified $V_{r}$ postulated to be within an upper branch range. Actual $V_{r}$ values at the peak $x-y$ responses are variable, depending on $m^{*}, \xi$ and Re. New 2-DOF VIV experimental studies which vary $\operatorname{Re}(\operatorname{Re}=V D / v)$ by altering $D$ (Blevins and Coughran, 2009) instead of $V$ (Stappenbelt et al., 2007) should be carried out to isolate the $V_{r}$ variation dependence from calibration, and to establish new empirical functions specifically for the peak $x\left(A^{*}\right)$ and $y\left(A^{*}\right)$ in place of the $A^{*}$ expression in Eq. (33) based on 1-DOF VIV.

(iv) A new realistic approximation of $\bar{C}_{D}$ is needed since its time-varying magnification gives rise to the 
increased hydrodynamic-added damping effect on overall response amplitudes through Eq. (11) and to the amplified in-line drift displacement through Eq. (9). Instead of using the typical 1-DOF expression in Eq (13), it would be more relevant to derive a new function depending on the above-mentioned $A^{*}{ }_{x}$ and $A^{*}$ as well as on a new best-fit coefficient $\bar{k}$ for 2-DOF VIV. For instance, Huera-Huarte and Bearman (2009) introduced two different $\bar{C}_{D}$ functions depending on individual $x$ and $y$ responses for a vertical flexible cylinder VIV.

(v) This study has fulfilled the aim of advancing the wake-cylinder oscillators by accounting for the effects of higher-order nonlinearities, $m^{*}$ and Re, and identifying the most influential variables, rather than achieving quantitative comparisons with experimental results which are, nonetheless, subject to some extra uncertainties of setup, measurement and post-processing. It is beyond our scope to justify whether those data used herein for calibration and deriving functions are more or less reliable. A sensitivity study treating a series of different experimental datasets would be meaningful as discussed, e.g., in Sarpkaya (2004).

(vi) The present wake oscillators and approaches can be applied to the modelling and sensitivity analysis of a three-dimensional VIV of long flexible cylinders (Zanganeh and Srinil, 2016). For such a multi-DOF system, a Galerkin-based modal reduction technique should be applied (Srinil, 2010, 2011; Srinil et al., 2009) prior to performing MCS in order to greatly reduce computational costs and data storage. This has recently been demonstrated in Low and Srinil (2016) for flexible risers undergoing cross-flow-only VIV.

\section{Conclusions}

Sensitivity studies of two-dimensional nonlinear wake-cylinder oscillators in predicting coupled cross-flow/inline VIV and in-line mean displacement amplifications of flexibly mounted rigid circular cylinders in uniform subcritical flows have been carried out, achieving an improved insight into the effects of empirical variables. The governing fluid-structure coupling terms have been advanced by accounting for the higher-order nonlinear effects of fluctuating lift-drag forces and steady-drag dynamic magnifications, depending on the relative flowcylinder velocities. The present phenomenological model captures some key features of two-degree-offreedom VIV comprising upper-branch responses with a jump, amplitude-dependent hydrodynamic damping, downstream drift, dual 2:1 resonance and figure-of-eight trajectory of $x$-y orbital motions, in addition to a pure in-line VIV. Based on experimental data in the literature, some existing and new empirical correlations have been introduced to incorporate a mass ratio and Re effect into the model coefficients.

A series of Monte Carlo simulations of nonlinear equations of coupled motions have been carried out to systematically and statistically examine the empirical sensitivity to the wake-cylinder oscillator variables affecting the two-dimensional fluid-structure interactions, maximum amplitudes and dynamically-amplified mean displacements. A low computational requirement of the present reduced-order model permits several thousands of simulations covering a wide range of system parameters. Complimentary numerical approaches, including scatterplots, Pearson's correlation coefficients, least-squares fits, regression analyses and Sobol' sensitivity indices, have been applied to qualitatively and quantitatively characterize individual contribution, relative importance, coupling and interdependence of multiple input variables influencing output uncertainties. A parametric range exhibiting a response jump with coexisting upper and lower branches is underlined as the most sensitive regime to the empirical variable alterations and initial conditions. 
Parametric results highlight that the two empirical coefficients of the lift force wake oscillator, with one governing the cylinder acceleration coupling term and the other regulating the wake damping, play the most influential role in the predictions of cross-flow/in-line amplitudes and steady-drag dynamic magnifications. This is sensible since the effective hydrodynamic added mass and damping characteristics depend on the fluid forces in phase with the cylinder accelerations and velocities, respectively. These wake oscillator variables are also strongly coupled with the cylinder geometric nonlinearities, especially for a low mass ratio system where greater two-dimensional amplitudes and amplified mean displacements are attained. Therefore, empirical input sensitivities and output uncertainties are amplitude-dependent. By accounting for the Re dependence through the hydrodynamic and wake oscillator coefficients, and performing a statistical hypothesis testing on model assumptions, the predicted maximum response in the case of higher mass ratio appears to be less sensitive, entailing a higher confidence level. It is also feasible to establish an optimal tuning coefficient via this statistical testing. The interdependence or correlation of empirical coefficients related to the coupled crossflow (maximum amplitude) and in-line (amplified drag coefficient) VIV is also meaningful. Numerical predictions can be overestimated or underestimated in a certain parametric randomness range when such a correlation effect is disregarded through the use of wake-cylinder oscillators.

The present sensitivity analyses provide an improved confidence for the modellers and tool developers in applying the wake-cylinder oscillators to predict cross-flow/in-line VIV. Overall empirical functions should be improved by incorporating more 2-DOF VIV datasets with controlled parameters to account for the multiple variable correlations. This key aspect should be addressed for such a phenomenological prediction model.

Acknowledgement: The authors thank anonymous reviewers and editors for their insightful comments which contribute to the quality improvement of this original manuscript.

\section{Appendix: Derivation of Structural Nonlinear Oscillators}

According to the Hamilton's principle (Meirovitch, 2001), the equations of two-dimensional motions of the four spring-mounted rigid cylinder shown in Fig. 1 can be derived, in a dimensional form, through

$$
\frac{d}{d t}\left(\frac{\partial \tilde{L}}{\partial \dot{u}_{j}}\right)-\frac{\partial \tilde{L}}{\partial u_{j}}=F_{j}-c_{s} \dot{u}_{j} \quad \text { for } j=1,2,
$$

where $\tilde{L}=T-P$ is the system Lagrangian, $T$ the kinetic energy, $P$ the potential energy, $u_{1}=\tilde{x}$ the in-line displacement, $u_{2}=\tilde{y}$ the cross-flow displacement, $F_{1}=F_{x}\left(F_{2}=F_{y}\right)$ the in-line (cross-flow) hydrodynamic force, $c_{s}$ the structural viscous damping coefficient, and $t$ the time. A dot denotes the derivative with respect to $t$.

By defining a generic stiffness coefficient $k$ of all springs with an original length $S$ at the initial static equilibrium state (point $O$ in Fig. 1), $T$ and $P$ associated with the oscillating cylinder are described by

$$
\begin{gathered}
T=\frac{1}{2} m\left(\dot{\tilde{x}}^{2}+\dot{\tilde{y}}^{2}\right), \\
P=\frac{k}{2}\left\{\left(\sqrt{(S-\tilde{x})^{2}+\tilde{y}^{2}}-S\right)^{2}+\left(\sqrt{(S+\tilde{x})^{2}+\tilde{y}^{2}}-S\right)^{2}+\left(\sqrt{\tilde{x}^{2}+(S-\tilde{y})^{2}}-S\right)^{2}+\left(\sqrt{\tilde{x}^{2}+(S+\tilde{y})^{2}}-S\right)^{2}\right\},
\end{gathered}
$$

where $m$ is the total system mass. By substituting Eqs. (A.2) and (A.3) into Eq. (A.1), the equations of cylinder motions can be obtained as 


$$
\begin{aligned}
& m \ddot{x}+c_{s} \dot{\tilde{x}}+4 k \tilde{x}+\frac{k(S-\tilde{x}) S}{\sqrt{(S-\tilde{x})^{2}+\tilde{y}^{2}}}-\frac{k(S+\tilde{x}) S}{\sqrt{(S+\tilde{x})^{2}+\tilde{y}^{2}}}-\frac{k \tilde{x} S}{\sqrt{\tilde{x}^{2}+(S-\tilde{y})^{2}}}-\frac{k \tilde{x} S}{\sqrt{\tilde{x}^{2}+(S+\tilde{y})^{2}}}=F_{x}, \\
& m \ddot{\tilde{y}}+c_{s} \dot{\tilde{y}}+4 k \tilde{y}+\frac{k(S-\tilde{y}) S}{\sqrt{\tilde{x}^{2}+(S-\tilde{y})^{2}}}-\frac{k(S+\tilde{y}) S}{\sqrt{\tilde{x}^{2}+(S+\tilde{y})^{2}}}-\frac{k \tilde{y} S}{\sqrt{(S-\tilde{x})^{2}+\tilde{y}^{2}}}-\frac{k \tilde{y} S}{\sqrt{(S+\tilde{x})^{2}+\tilde{y}^{2}}}=F_{y} .
\end{aligned}
$$

The above system equations are highly nonlinear and coupled, which are impractical to solve directly. To simplify this complexity, the nonlinear geometric terms may be expanded by using the two-variable Taylor series expansion with respect to the initial static position $(\tilde{x}=0, \tilde{y}=0)$. Accordingly, each nonlinear term in Eqs. (A.4) and (A.5) is approximated, by keeping the series to the third order with the aim of capturing a coupling of cross-flow/in-line displacements associated with the coupled lift-drag hydrodynamics, as

$$
\begin{aligned}
\frac{(S-\tilde{x})}{\sqrt{(S-\tilde{x})^{2}+\tilde{y}^{2}}} \approx 1-\frac{\tilde{y}^{2}}{2 S^{2}}-\frac{\tilde{x} \tilde{y}^{2}}{S^{3}}, & \frac{(S+\tilde{x})}{\sqrt{(S+\tilde{x})^{2}+\tilde{y}^{2}}} \approx 1-\frac{\tilde{y}^{2}}{2 S^{2}}+\frac{x \tilde{y}^{2}}{S^{3}}, \\
\frac{\tilde{x}}{\sqrt{\tilde{x}^{2}+(S-\tilde{y})^{2}}} \approx \frac{\tilde{x}}{S}+\frac{\tilde{x} \tilde{y}}{S^{2}}-\frac{\tilde{x}^{3}}{2 S^{3}}+\frac{\tilde{x} \tilde{y}^{2}}{S^{3}}, & \frac{\tilde{x}}{\sqrt{\tilde{x}^{2}+(S+\tilde{y})^{2}}} \approx \frac{\tilde{x}}{S}-\frac{\tilde{x} \tilde{y}}{S^{2}}-\frac{\tilde{x}^{3}}{2 S^{3}}+\frac{\tilde{x} \tilde{y}^{2}}{S^{3}}, \\
\frac{(S-\tilde{y})}{\sqrt{\tilde{x}^{2}+(S-\tilde{y})^{2}}} \approx 1-\frac{\tilde{x}^{2}}{2 S^{2}}-\frac{x^{2} \tilde{y}}{S^{3}}, & \frac{(S+\tilde{y})}{\sqrt{\tilde{x}^{2}+(S+\tilde{y})^{2}}} \approx 1-\frac{\tilde{x}^{2}}{2 S^{2}}+\frac{x^{2} \tilde{y}}{S^{3}}, \\
\frac{\tilde{y}}{\sqrt{(S-\tilde{x})^{2}+\tilde{y}^{2}}} \approx \frac{\tilde{y}}{S}+\frac{\tilde{x} \tilde{y}}{S^{2}}-\frac{\tilde{y}^{3}}{2 S^{3}}+\frac{\tilde{x}^{2} \tilde{y}}{S^{3}}, & \frac{\tilde{y}}{\sqrt{(S+\tilde{x})^{2}+\tilde{y}^{2}}} \approx \frac{\tilde{y}}{S}-\frac{\tilde{x} \tilde{y}}{S^{2}}-\frac{\tilde{y}^{3}}{2 S^{3}}+\frac{\tilde{x}^{2} \tilde{y}}{S^{3}} .
\end{aligned}
$$

By substituting Eq. (A.6) into (A.4), and Eq. (A.7) into (A.5), the quadratic nonlinear terms ( $\tilde{x} \tilde{y}, \tilde{x}^{2}, \tilde{y}^{2}$ ) are effectively cancelled out. By letting $K_{x}=K_{y}=2 k$ and $\tilde{s}=\left(1 / 2 S^{2}\right)$, the ensuing equations of motion read

$$
\begin{aligned}
m \ddot{\tilde{x}}+c_{s} \dot{\tilde{x}}+K_{x}\left(\tilde{x}+\tilde{s} \tilde{x}^{3}-4 \tilde{s} \tilde{y} \tilde{y}^{2}\right) & =F_{x}, \\
m \ddot{\tilde{y}}+c_{s} \dot{\tilde{y}}+K_{y}\left(\tilde{y}+\tilde{s} \tilde{y}^{3}-4 \tilde{s} \tilde{y} \tilde{x}^{2}\right) & =F_{y} .
\end{aligned}
$$

This dynamic system contains cubic nonlinear terms $\left(\tilde{x}^{3}, \tilde{y}^{3}, \tilde{x} \tilde{y}^{2}, \tilde{y} \tilde{x}^{2}\right)$ which may produce a hardening $(+)$ or softening (-) effect. The variable $\tilde{s}$ is arbitrary, depending on $S$. Higher-order nonlinear terms $(n>3)$ with \pm coefficients become small and negligible due to the multiplication of $1 / S^{n-1}$ fractions. If $\tilde{s}$ is known, e.g., as in the experiment, Eqs. (A.8) and (A.9) may be used. The hydrodynamic excitations $F_{x}$ and $F_{y}$ can be evaluated using the computational fluid dynamics simulation based on the Navier-Stokes equations.

Nevertheless, for a phenomenological fluid-cylinder interaction modelling involving the van der Pol wake oscillators, Eqs. (6) and (8), approximating $F_{x}$ and $F_{y}, \tilde{s}$ is arbitrary and the cubic nonlinear coefficients may be assumed, together with the wake coefficients $\left(\varepsilon_{x}, \Lambda_{x}, \varepsilon_{y}, \Lambda_{y}\right)$, towards the purpose of VIV response calibration and prediction. Following Srinil and Zanganeh (2012), empirical tuning parameters $\left(\tilde{\alpha}_{x}, \tilde{\beta}_{x}, \tilde{\alpha}_{y}, \tilde{\beta}_{y}\right)$ may be introduced to replace unknowns $\tilde{s}$ and $-4 \tilde{s}$. By letting $K_{x}=K_{y}=K$ for the two-dimensionally equal natural frequencies, Eqs. (A.8) and (A.9) may be alternatively written as

$$
\begin{aligned}
& m \ddot{\tilde{x}}+c_{s} \dot{\tilde{x}}+K\left(\tilde{x}+\tilde{\alpha}_{x} \tilde{x}^{3}+\tilde{\beta}_{x} \tilde{x} \tilde{y}^{2}\right)=F_{x}, \\
& m \ddot{\tilde{y}}+c_{s} \dot{\tilde{y}}+K\left(\tilde{y}+\tilde{\alpha}_{y} \tilde{y}^{3}+\tilde{\beta}_{y} \tilde{y} \tilde{x}^{2}\right)=F_{y} .
\end{aligned}
$$

These equations, together with the added mass and projected excitation forces, are presented by Eqs. (1) and (2). Through a normalisation procedure with respect to $D$ for the displacements and $\omega_{n}$ for the time, the 
dimensionless equations of cylinder motions are expressed by Eqs. (5) and (7) with the dimensionless variables $\alpha_{x}, \beta_{x}, \alpha_{y}$ and $\beta_{y}$, dependent on the factor $D^{2} / S^{2}$. Substantial calibration of coupled cylinder-wake oscillators, Eqs. (5)-(8), with experimental data of Jauvtis and Williamson (2004), Blevins and Coughran (2009), and Stappenbelt et al. (2007), and a series of parametric studies, have been performed by Srinil and Zanganeh (2012) who suggested $\alpha_{x}=\beta_{x}=\alpha_{y}=\beta_{y}=0.7$ in combination with other empirical variables reported in Table 2 .

To justify the use of Eqs. (A.10) and (A.11), the predicted 2-DOF VIV amplitudes based on Eqs. (5)-(8) in the varying $V_{r}$ cases are presented in Fig. A1 with $m^{*}=3.68$ and $\xi=0.006$ associated with Fig. 5. Experimental data of Stappenbelt et al. (2007) are used for a tuning and comparison. Three cases are shown: (a) the linear model with $\alpha_{x}=\beta_{x}=\alpha_{y}=\beta_{y}=0$; (b) the nonlinear model with $\alpha_{x}=\alpha_{y}=0.175$ and $\beta_{x}=\beta_{y}=-0.7$ (i.e., $\tilde{\alpha}_{x}=\tilde{\alpha}_{y}=\tilde{s}$ and $\tilde{\beta}_{x}=\tilde{\beta}_{y}=-4 \tilde{s}$ as in Eqs. A.8 and A.9), and (c) the proposed nonlinear model with $\alpha_{x}=\beta_{x}=\alpha_{y}=\beta_{y}=0.7$. With $\alpha_{x}=\alpha_{y}=0.175$ and $\beta_{x}=\beta_{y}=-0.7$, the predicted maximum $A_{x} / D$ is considerably overestimated and there is a left shift in both $A_{x} / D$ and $A_{y} / D$ peaks to a lower $V_{r}$. This shift also appears when considering the linear model which underestimates $A_{x} / D$. For these models (a) and (b), the predicted responses appear to be continuous with a narrow upper-branch range without showing a response jump or discontinuity, unlike the experimental response displaying a hardening-type feature with a downward jump as $V_{r}$ is increased. With $\alpha_{x}=\beta_{x}=\alpha_{y}=\beta_{y}=0.7$, both a downward and upward jump (vertical dashed lines) is captured when increasing and decreasing $V_{r}$ in both $A_{x} / D$ and $A_{y} / D$ diagrams, showing an improved prediction of $X-Y$ hardening response, amplitudes and peak locations vs. experimental data. These comparisons suggest that, despite the van der Pol wake oscillators cannot capture the exact fluid mechanics and hydrodynamic effects, the 2-DOF VIV predictions may be improved by accounting for cubic nonlinear terms and empirically-tuned coefficients in structural oscillators.

In this study, Eqs. (5)-(8) are used as a generic phenomenological prediction model for the comprehensive empirical sensitivity analyses to demonstrate the input-output variability in VIV and amplified mean response predictions based on such coupled nonlinear wake-cylinder oscillators.
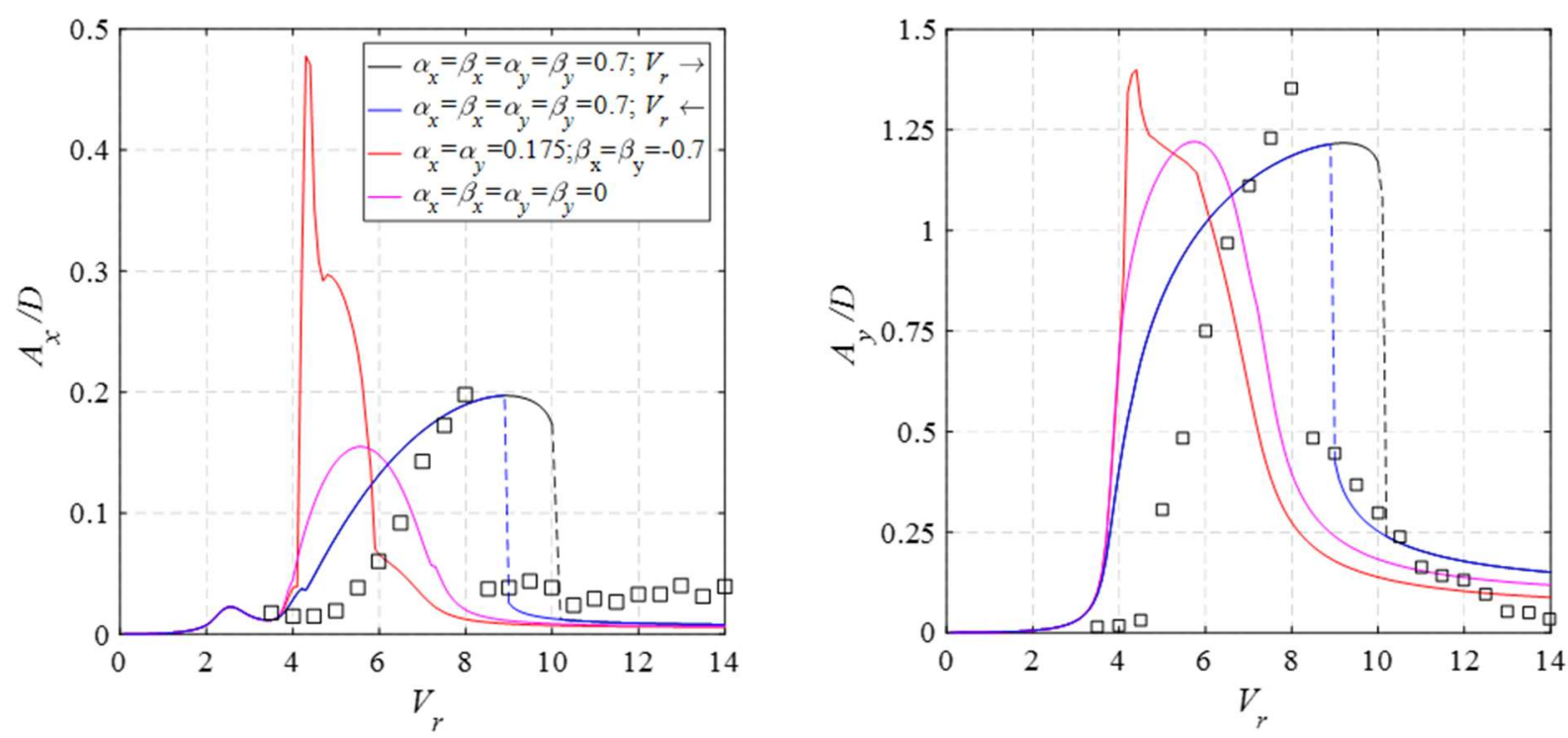

Figure A1 Comparisons of response amplitudes based on different nonlinear and linear cylinder models, and experimental data (squares) of Stappenbelt et al. (2007) with $m^{*}=3.68$ and $\xi=0.006$. 


\section{References}

Bearman, P.W., 2011. Circular cylinder wakes and vortex-induced vibrations. Journal of Fluids and Structures 27, 648-658.

Bishop, R.E.D., Hassan, A.Y., 1964a. The lift and drag forces on a circular cylinder in a flowing fluid. Proceedings of the Royal Society of London. Series A. 277, 32-50.

Bishop, R.E.D., Hassan, A.Y., 1964b. The lift and drag forces on a circular cylinder oscillating in a flowing fluid. Proceedings of the Royal Society of London. Series A. 277, 51-75.

Bitner-Gregersen, E.M., Hagen, O., 1990. Uncertainties in data for the offshore environment. Structural Safety 7, 11-34.

Blevins, R.D., 1990. Flow-Induced Vibrations. Van Nostrand Reinhold, New York.

Blevins, R.D., Coughran, C.S., 2009. Experimental investigation of vortex-induced vibration in one and two dimensions with variable mass, damping, and Reynolds number. Journal of Fluids Engineering 131, 101202101207.

Cannavó, F., 2012. Sensitivity analysis for volcanic source modeling quality assessment and model selection. Computers \& Geosciences 44, 52-59.

Cattarin, G., Pagliano, L., Causone, F., Kindinis, A., Goia, F., Carlucci, S., Schlemminger, C., 2018. Empirical validation and local sensitivity analysis of a lumped-parameter thermal model of an outdoor test cell. Building and Environment 130, 151-161.

Cheung, J.C.K., Melbourne, W.H., 1983. Turbulence effects on some aerodynamic parameters of a circular cylinder at supercritical numbers. Journal of Wind Engineering and Industrial Aerodynamics 14, 399-410.

Currie, I.G., Turnbull, D.H., 1987. Streamwise oscillations of cylinders near the critical Reynolds number. Journal of Fluids and Structures 1, 185-196.

Dahl, J.M., Hover, F.S., Triantafyllou, M.S., Oakley, O.H., 2010. Dual resonance in vortex-induced vibrations at subcritical and supercritical Reynolds numbers. Journal of Fluid Mechanics 643, 395-424.

DNV, 2010. DNV-RP-F204 Riser Fatigue.

Draper, N.R., Smith, H., 1981. Applied Regression Analysis. Wiley, New York.

Facchinetti, M.L., de Langre, E., Biolley, F., 2004. Coupling of structure and wake oscillators in vortexinduced vibrations. Journal of Fluids and Structures 19, 123-140.

Franzini, G.R., Bunzel, L.O., 2018. A numerical investigation on piezoelectric energy harvesting from vortexinduced vibrations with one and two degrees of freedom. Journal of Fluids and Structures 77, 196-212.

Gabbai, R.D., Benaroya, H., 2005. An overview of modeling and experiments of vortex-induced vibration of circular cylinders. Journal of Sound and Vibration 282, 575-616.

Gabbai, R.D., Hiebert, J., 2011. Sensitivity analysis of a generic wake-body model for the vortex-induced vibration of a rigid circular cylinder using Monte Carlo simulations, The 30th International Conference on Offshore Mechanics and Arctic Engineering, pp. 91-96.

Govardhan, R., Williamson, C., 2006. Defining the 'modified Griffin plot' in vortex-induced vibration: revealing the effect of Reynolds number using controlled damping. Journal of Fluid Mechanics 561, 147-180. Huera-Huarte, F.J., Bearman, P.W., 2009. Wake structures and vortex-induced vibrations of a long flexible 
cylinder-Part 2: Drag coefficients and vortex modes. Journal of Fluids and Structures 25, 991-1006.

Iwan, W.D., Blevins, R.D., 1974. A model for vortex induced oscillation of structures. ASME Journal of Applied Mechanics 41, 581-585.

Jauvtis, N., Williamson, C.H.K., 2004. The effect of two degrees of freedom on vortex-induced vibration at low mass and damping. Journal of Fluid Mechanics 509, 23-62.

King, R., 1977. A review of vortex shedding research and its application. Ocean Engineering 4, 141-171.

Krenk, S., Nielsen, S.R.K., 1999. Energy balanced double oscillator model for vortex-induced vibrations. Journal of Engineering Mechanics 125, 263-271.

Krzywinski, M., Altman, N., 2013. Significance, P values and t-tests. Nature Methods 10, 1041.

Lie, H., Kaasen, K.E., 2006. Modal analysis of measurements from a large-scale VIV model test of a riser in linearly sheared flow. Journal of Fluids and Structures 22, 557-575.

Lie, H., Larsen, C.M., Kaasen, K.E., 2008. Frequency domain model for prediction of stochastic vortex induced vibrations for deep water risers, The 27th International Conference on Offshore Mechanics and Arctic Engineering, Portugal, pp. 809-821.

Low, Y.M., Srinil, N., 2016. VIV fatigue reliability analysis of marine risers with uncertainties in the wake oscillator model. Engineering Structures 106, 96-108.

Lucor, D., Su, C.H., Karniadakis, G.E., 2004. Generalized polynomial chaos and random oscillators. International Journal for Numerical Methods in Engineering 60, 571-596.

Lucor, D., Xiu, D., Su, C.H., Karniadakis, G.E., 2003. Predictability and uncertainty in CFD. International Journal for Numerical Methods in Fluids 43, 483-505.

Ma, B., Srinil, N., 2017. Two-dimensional vortex-induced vibration suppression through the cylinder transverse linear/nonlinear velocity feedback. Acta Mechanica, in press.

Ma, J.Z., Ackerman, E., Yang, J.-J., 1993. Parameter sensitivity of a model of viral epidemics simulated with monte carlo techniques. I. Illness attack rates. International Journal of Bio-Medical Computing 32, 237-253. Meirovitch, L., 2001. Fundamentals of Vibrations. McGraw-Hill.

Melchers, R.E., 1999. Structural Reliability Analysis and Prediction. John Wiley \& Sons.

Modarres-Sadeghi, Y., Hover, F.S., Triantafyllou, M.S., 2008. Fatigue calculation of risers using a van der Pol wake oscillator model with random parameters, The 27th International Conference on Offshore Mechanics and Arctic Engineering, pp. 757-763.

Mukundan, H., Hover, F.S., Triantafyllou, M.S., 2010. A systematic approach to riser VIV response reconstruction. Journal of Fluids and Structures 26, 722-746.

Norberg, C., 2003. Fluctuating lift on a circular cylinder: review and new measurements. Journal of Fluids and Structures 17, 57-96.

Ogink, R.H.M., Metrikine, A.V., 2010. A wake oscillator with frequency dependent coupling for the modeling of vortex-induced vibration. Journal of Sound and Vibration 329, 5452-5473.

Opinel, P.-A., Srinil, N., 2018. Phenomenological modelling of cylinder VIV with contributions from oscillatory flows, The 37th International Conference on Ocean, Offshore and Arctic Engineering, Madrid, pp. OMAE2018-77689. 
Paidoussis, M.P., Price, S.J., de Langre, E., 2011. Fluid-Structure Interactions: Cross-Flow-Induced Instabilities. Cambridge University Press.

Saltelli, A., Chan, K., Scott, E.M., 2000. Sensitivity Analysis. John Wiley \& Sons.

Sanchis, A., Sælevik, G., Grue, J., 2008. Two-degree-of-freedom vortex-induced vibrations of a springmounted rigid cylinder with low mass ratio. Journal of Fluids and Structures 24, 907-919.

Sarpkaya, T., 2004. A critical review of the intrinsic nature of vortex-induced vibrations. Journal of Fluids and Structures 19, 389-447.

Schewe, G., 1983. On the force fluctuations acting on a circular cylinder in crossflow from subcritical up to transcritical Reynolds numbers. Journal of Fluid Mechanics 133, 265-285.

Schlichting, H., Gersten, K., 2017. Boundary-Layer Theory. Springer, Berlin.

Skop, R.A., Balasubramanian, S., 1997. A new twist on an old model for vortex-excited vibrations. Journal of Fluids and Structures 11, 395-412.

Sobol, I.M., 2001. Global sensitivity indices for nonlinear mathematical models and their Monte Carlo estimates. Mathematics and Computers in Simulation 55, 271-280.

Srinil, N., 2010. Multi-mode interactions in vortex-induced vibrations of flexible curved/straight structures with geometric nonlinearities. Journal of Fluids and Structures 26, 1098-1122.

Srinil, N., 2011. Analysis and prediction of vortex-induced vibrations of variable-tension vertical risers in linearly sheared currents. Applied Ocean Research 33, 41-53.

Srinil, N., Ma, B., Zhang, L., 2018. Experimental investigation on in-plane/out-of-plane vortex-induced vibrations of curved cylinder in parallel and perpendicular flows. Journal of Sound and Vibration 421, 275 299.

Srinil, N., Wiercigroch, M., O’Brien, P., 2009. Reduced-order modelling of vortex-induced vibration of catenary riser. Ocean Engineering 36, 1404-1414.

Srinil, N., Zanganeh, H., 2012. Modelling of coupled cross-flow/in-line vortex-induced vibrations using double Duffing and van der Pol oscillators. Ocean Engineering 53, 83-97.

Srinil, N., Zanganeh, H., Day, A., 2013. Two-degree-of-freedom VIV of circular cylinder with variable natural frequency ratio: Experimental and numerical investigations. Ocean Engineering 73, 179-194.

Stappenbelt, B., Lalji, F., Tan, G., 2007. Low mass ratio vortex-induced motion, The 16th Australasian Fluid Mechanics Conference, Gold Coast, Australia,, pp. 1491-1497.

Stappenbelt, B., O'Neill, L., 2007. Vortex-induced vibration of cylindrical structures with low mass ratio, The 17th International Offshore and Polar Engineering Conference, Lisbon.

Sumer, B.M., Fredsoe, J., 2006. Hydrodynamics Around Cylindrical Structures. World Scientific.

Ulveseter, J.V., Thorsen, M.J., Sævik, S., Larsen, C.M., 2017. Stochastic modelling of cross-flow vortexinduced vibrations. Marine Structures 56, 260-280.

Vandiver, J.K., Jong, J.Y., 1987. The relationship between in-line and cross-flow vortex-induced vibration of cylinders. Journal of Fluids and Structures 1, 381-399.

Williamson, C.H.K., Govardhan, R., 2004. Vortex-induced vibrations. Annual Review of Fluid Mechanics 36, 413-455. 
Wu, X., Ge, F., Hong, Y., 2012. A review of recent studies on vortex-induced vibrations of long slender cylinders. Journal of Fluids and Structures 28, 292-308.

Xiu, D., Lucor, D., Su, C.H., Karniadakis, G.E., 2002. Stochastic modeling of flow-structure interactions using generalized polynomial chaos. Journal of Fluids Engineering 124, 51-59.

Yang, W., Ai, Z., Zhang, X., Chang, X., Gou, R., 2018. Nonlinear dynamics of three-dimensional vortexinduced vibration prediction model for a flexible fluid-conveying pipe. International Journal of Mechanical Sciences 138-139, 99-109.

Zanganeh, H., Srinil, N., 2014. Characterization of variable hydrodynamic coefficients and maximum responses in two-dimensional vortex-induced vibrations with dual resonances. Journal of Vibration and Acoustics 136, 051010.

Zanganeh, H., Srinil, N., 2016. Three-dimensional VIV prediction model for a long flexible cylinder with axial dynamics and mean drag magnifications. Journal of Fluids and Structures 66, 127-146.

Zdravkovich, M.M., 1997. Flow Around Circular Cylinders Volume I: Fundamentals. Oxford Science Press. 


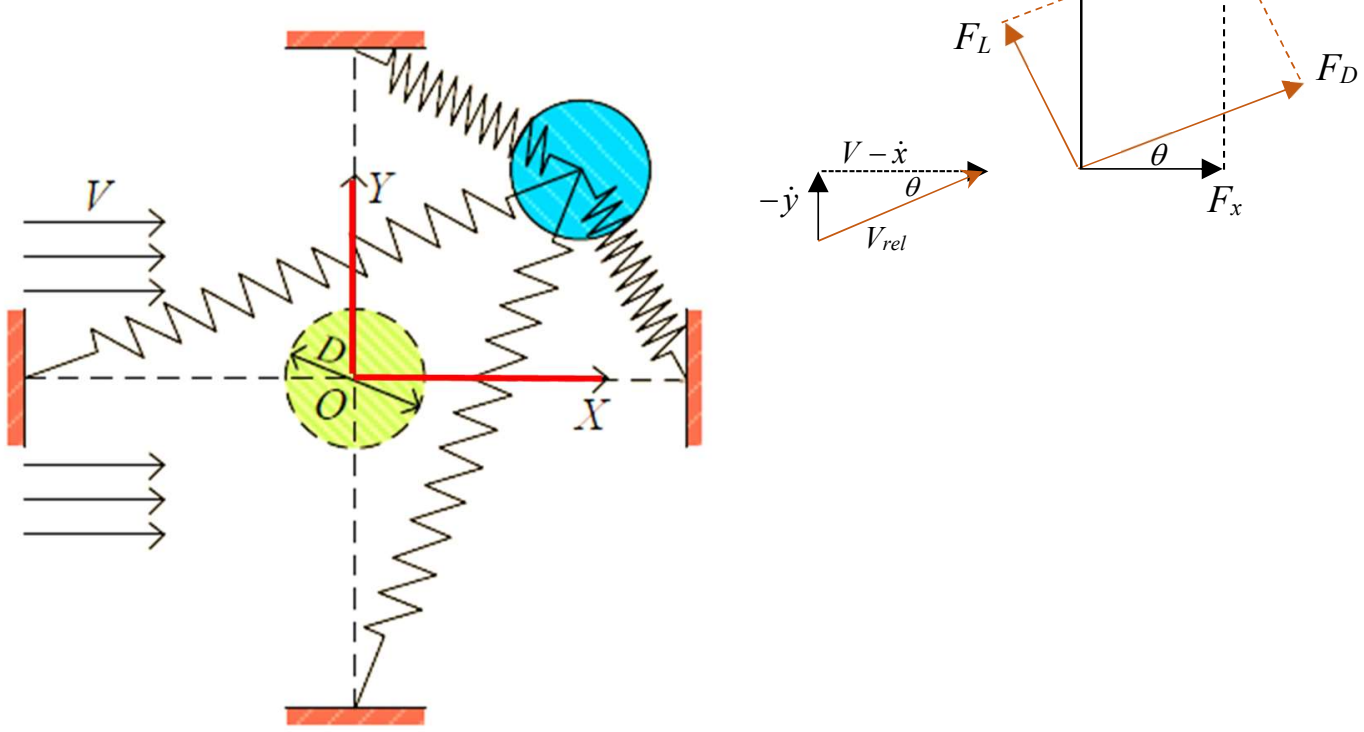

Figure 1 A schematic diagram of cross-flow/in-line VIV of an elastically mounted circular cylinder in uniform flows, vectors of the relative flow-cylinder velocities and associated hydrodynamic force components. 
a)

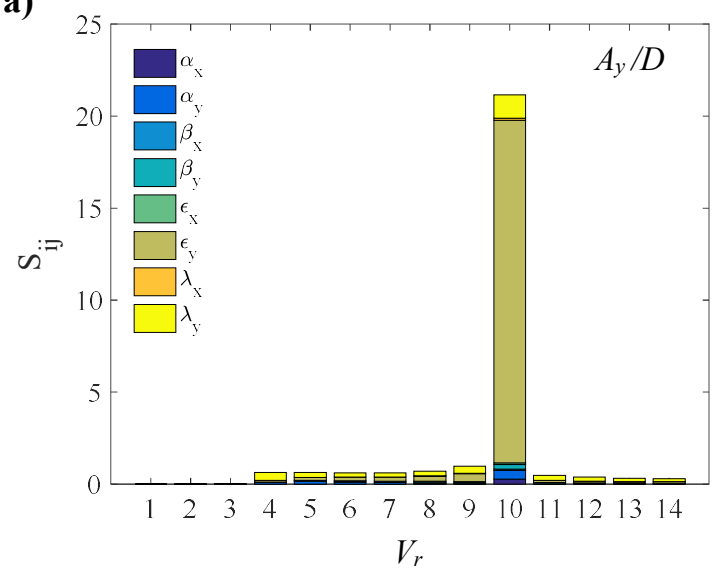

b)

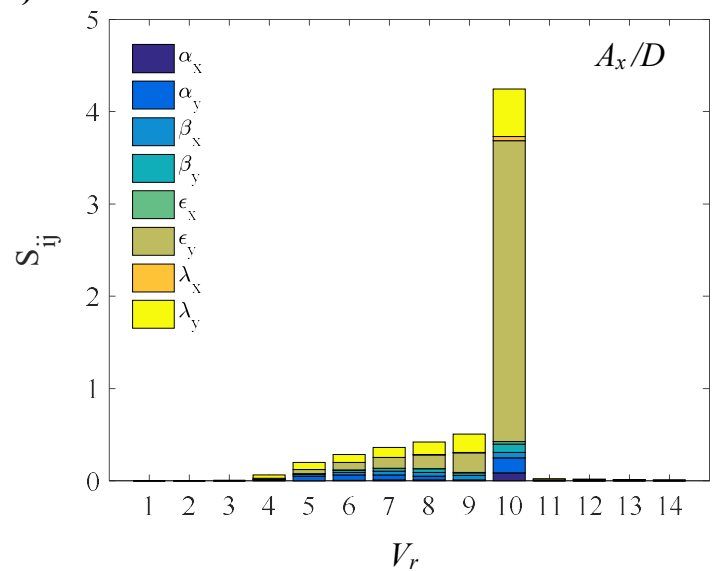

c)

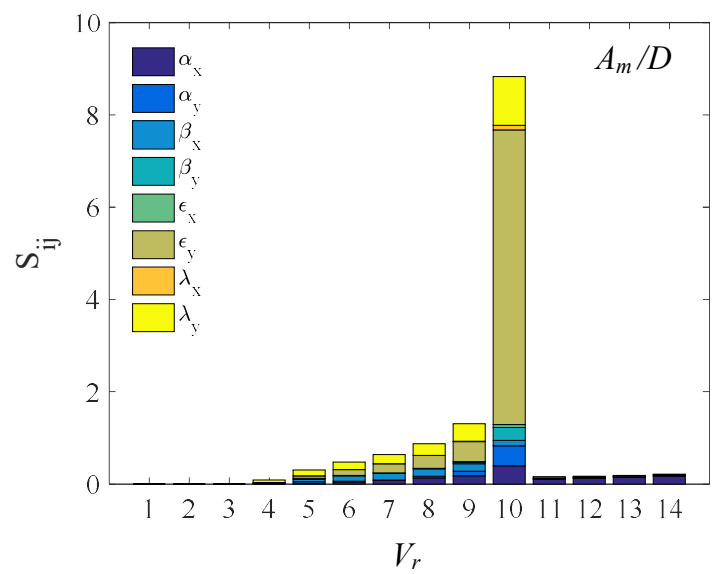

d)

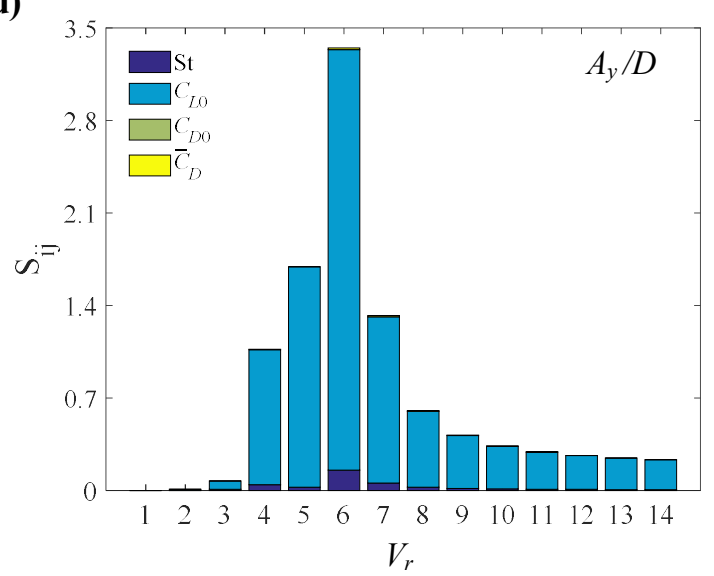

e)

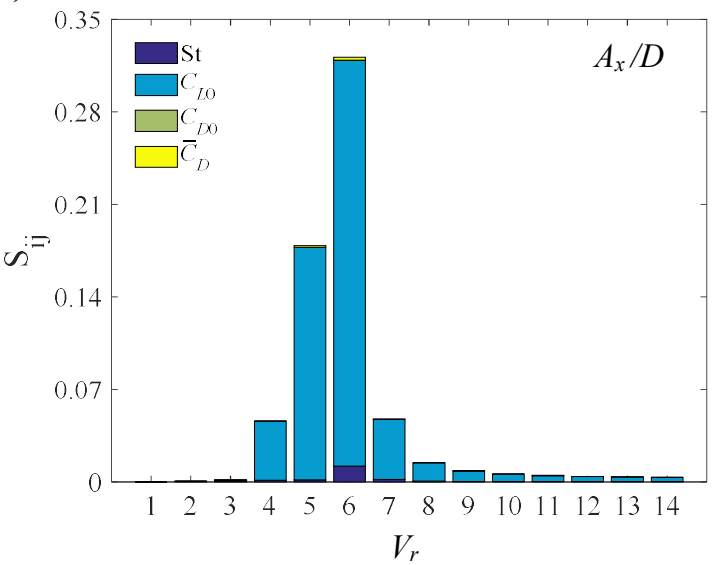

f)

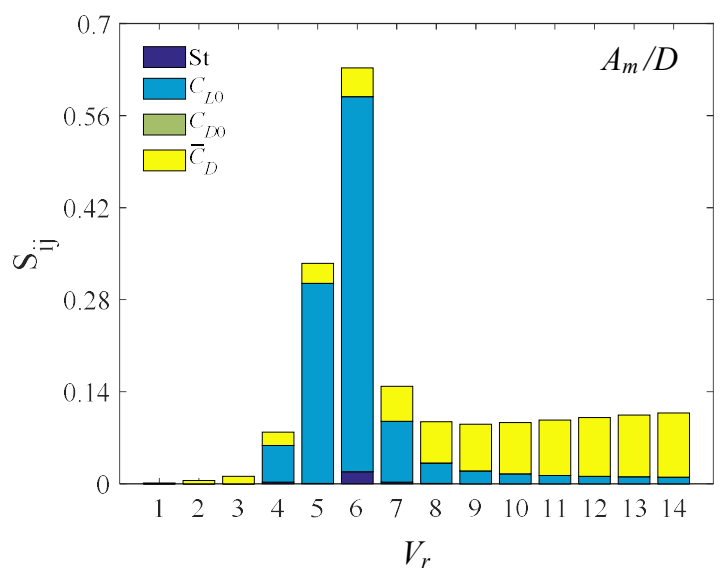

Figure 2 Local sensitivity indices of response outputs for cylinder with $m^{*}=3.68$ and $\xi=0.006$ due to perturbation in (a-c) wake-cylinder oscillator and (d-f) hydrodynamic coefficients. 
a)

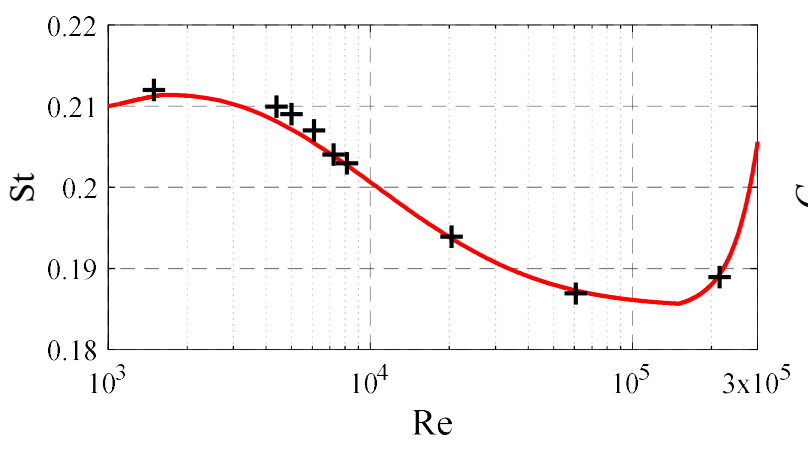

c)

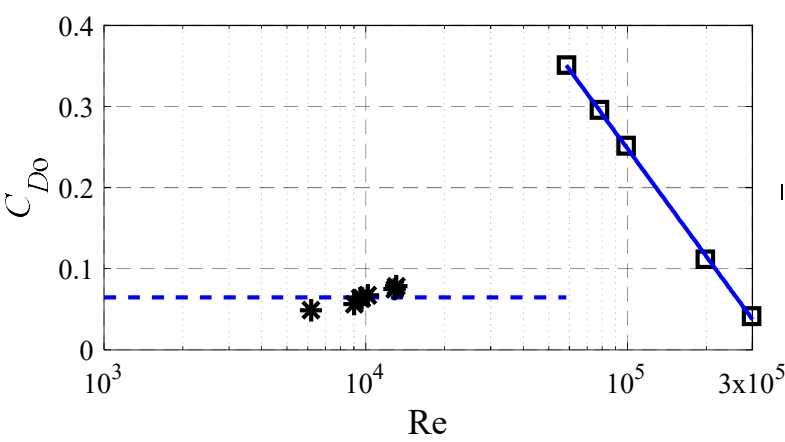

b)

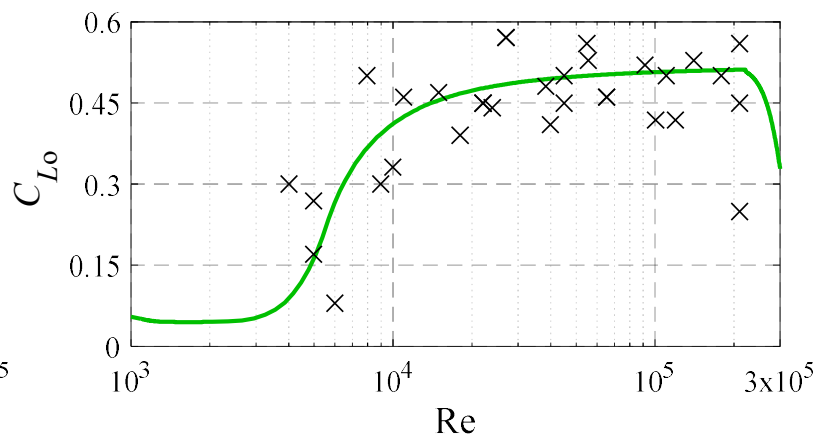

d)

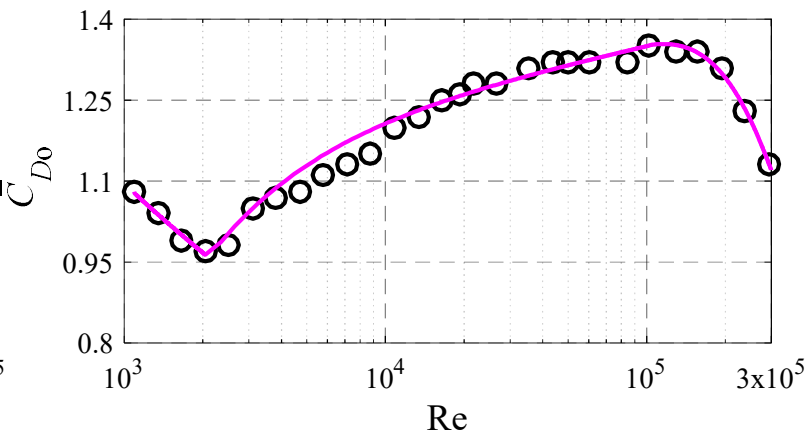

Figure 3 Variation of hydrodynamic coefficients as function of Re for a stationary cylinder: star, square and circular symbols denote test data from Bishop and Hassan (1964a), Cheung and Melbourne (1983), and Schlichting and Gersten (2017), respectively; solid lines in a)-d) are based on Eqs. (22)-(32); some test data (plus and cross symbols) in a) and b) are from Norberg (2003); a dashed line in c) denotes a mean value. 
a)

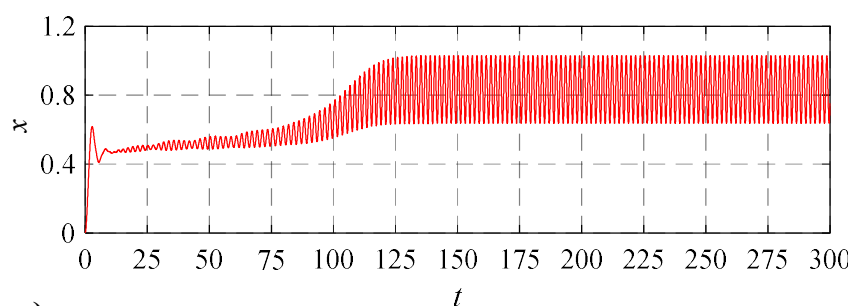

c)

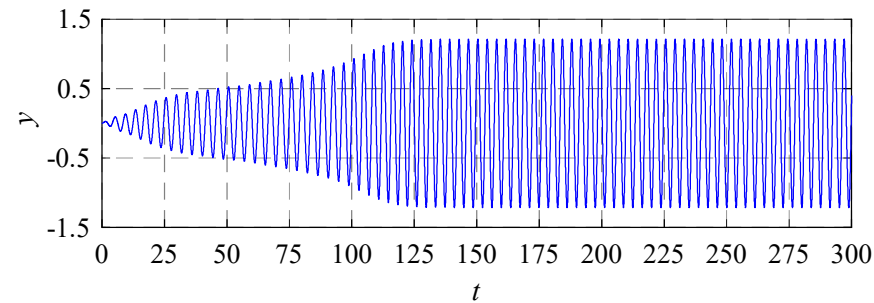

b)

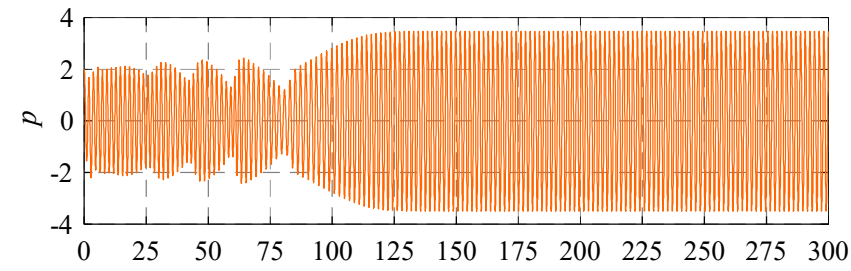

d)

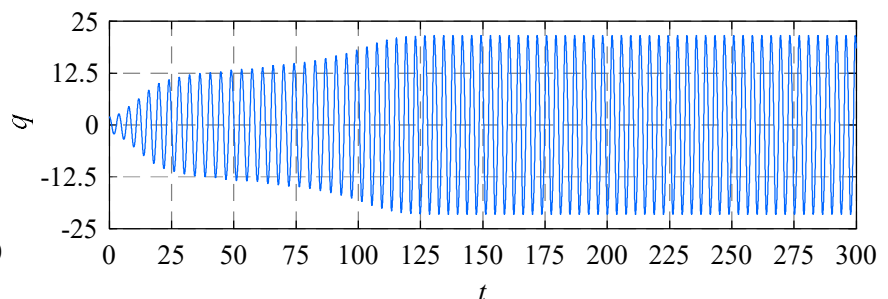

Figure 4 Illustrative time histories of four wake-cylinder variables in 2-DOF cross-flow/in-line VIV with $m^{*}=3.68, \xi=0.006$ and $V_{r}=9$. 
a)
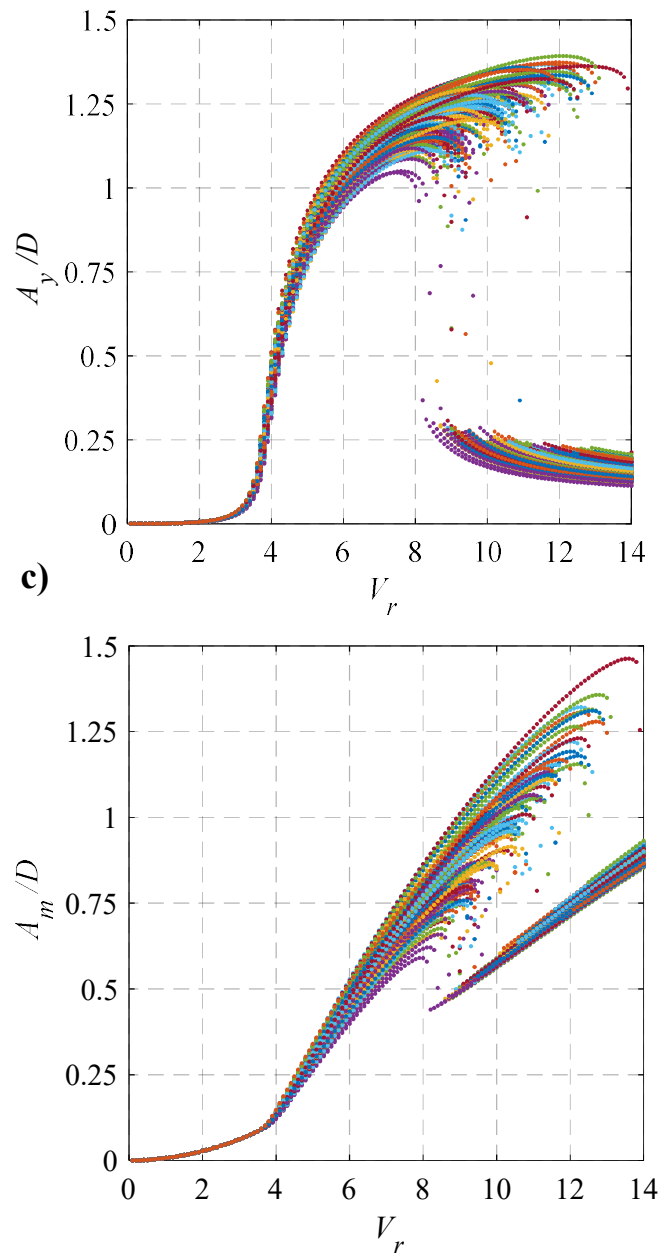

b)
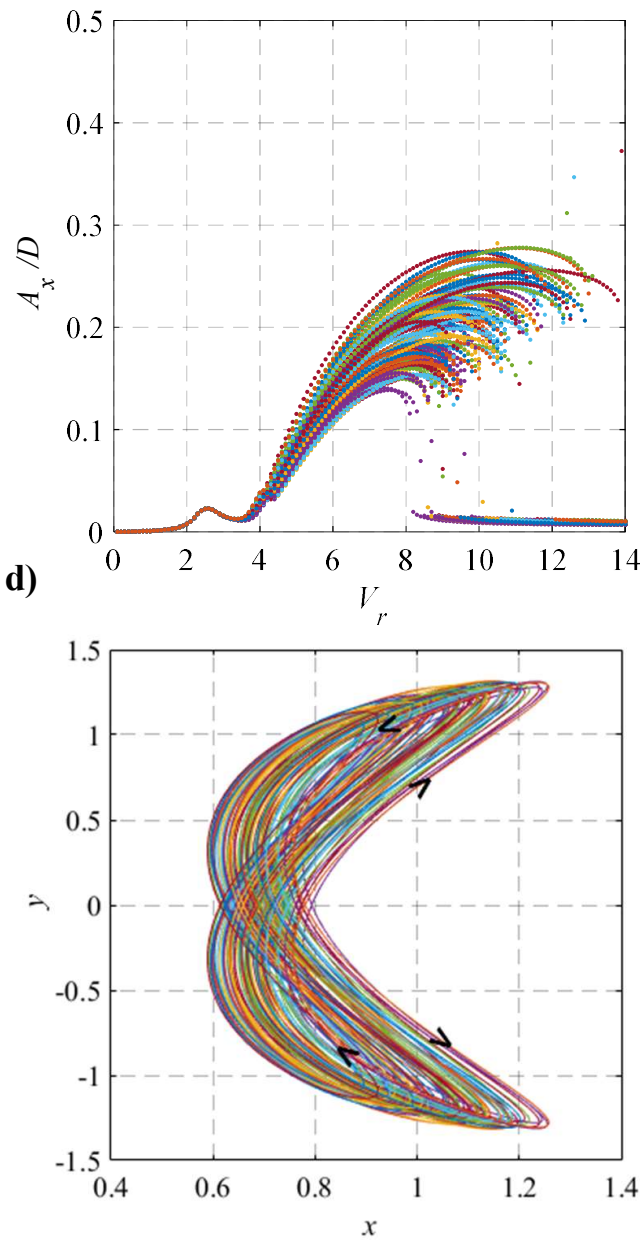

Figure 5 Illustrative (a) cross-flow and (b) in-line responses, (c) in-line mean displacements and (d) $x-y$ trajectories at $V_{r}=9$, for cylinder with $m^{*}=3.68, \xi=0.006$, and sample 100 MCS for each $V_{r}$. 
a)

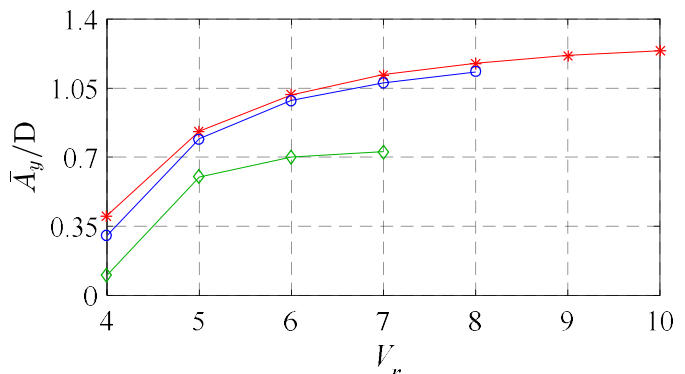

c)

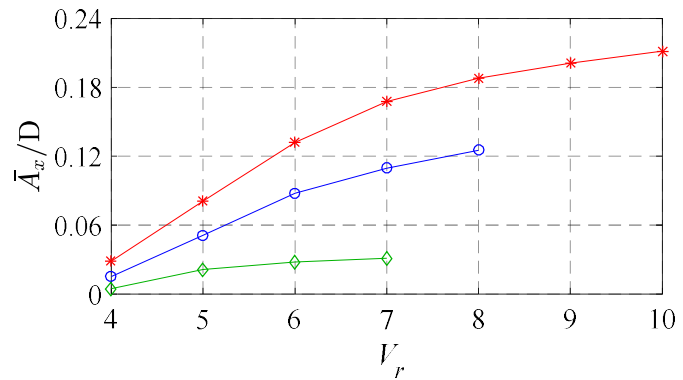

e)

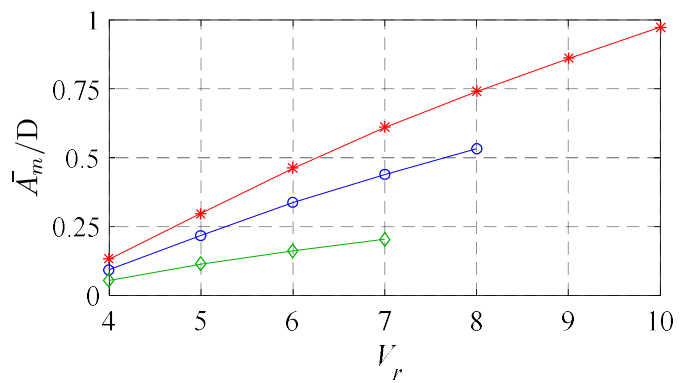

b)

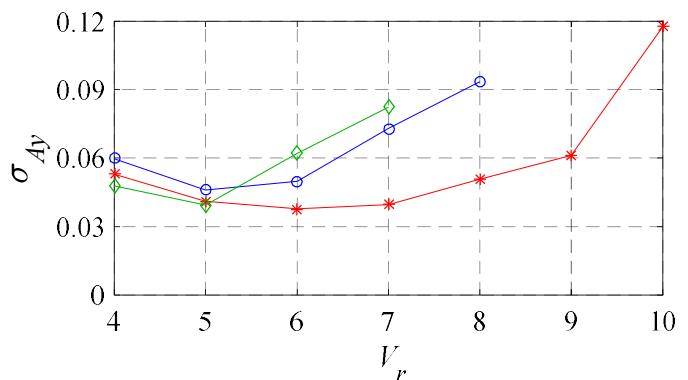

d)

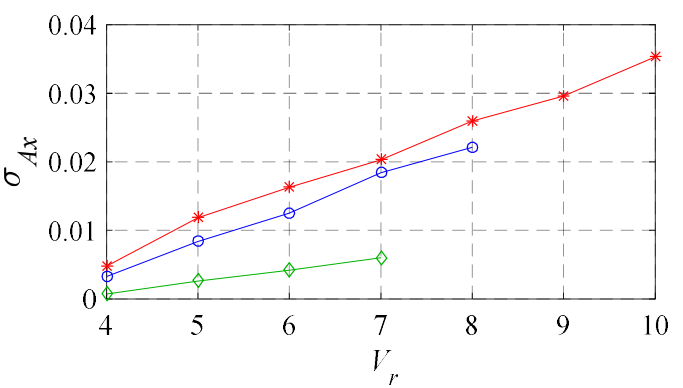

f)

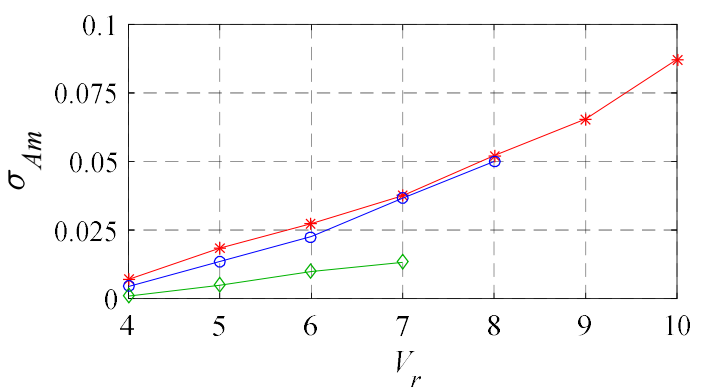

Figure 6 Mean (a, c, e) and standard deviation (b, d, f) values of $A_{y} / D, A_{x} / D$ and $A_{m} / D$ based on 1000 MCS for different $V_{r}$ and $m^{*}$ : star, circle and diamond symbols denote $m^{*}=3.68,5.19$ and 8.76, respectively. 
a)
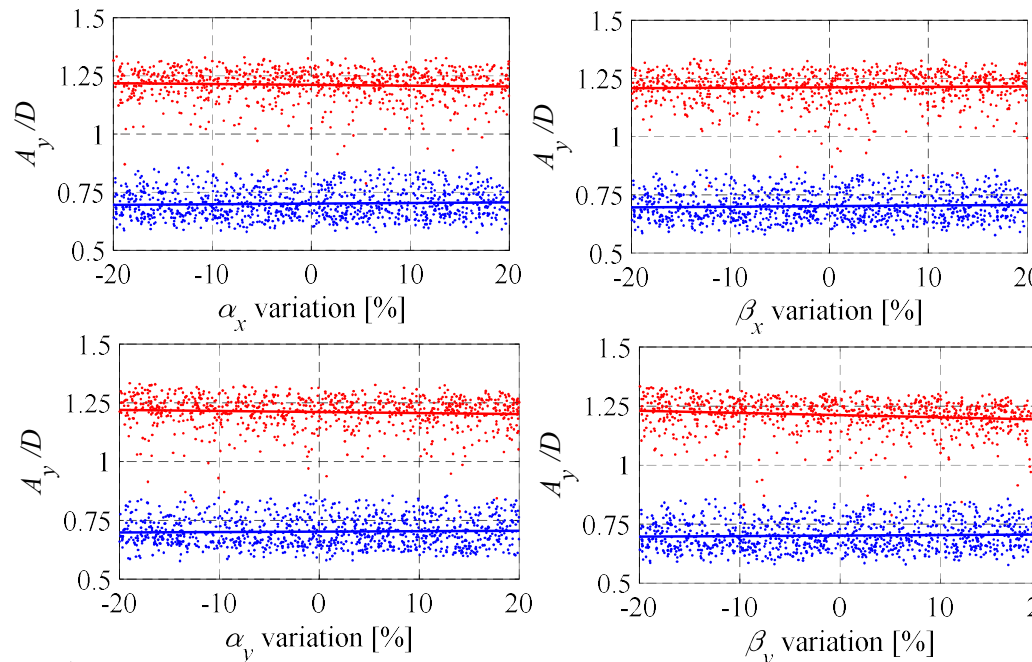

b)

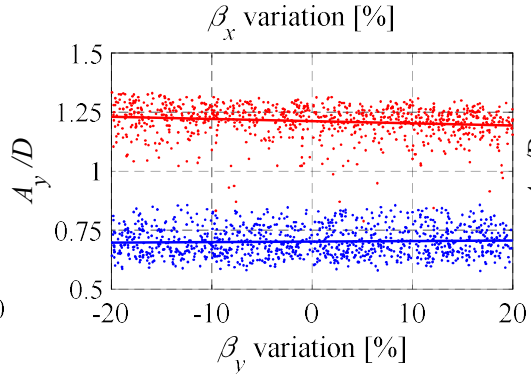

c)
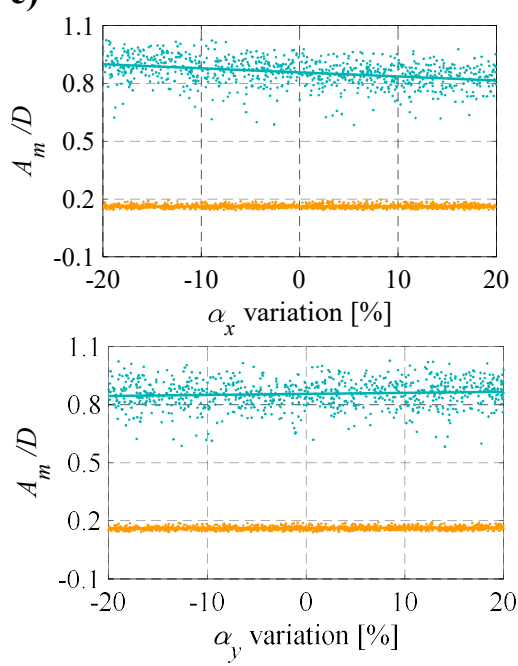
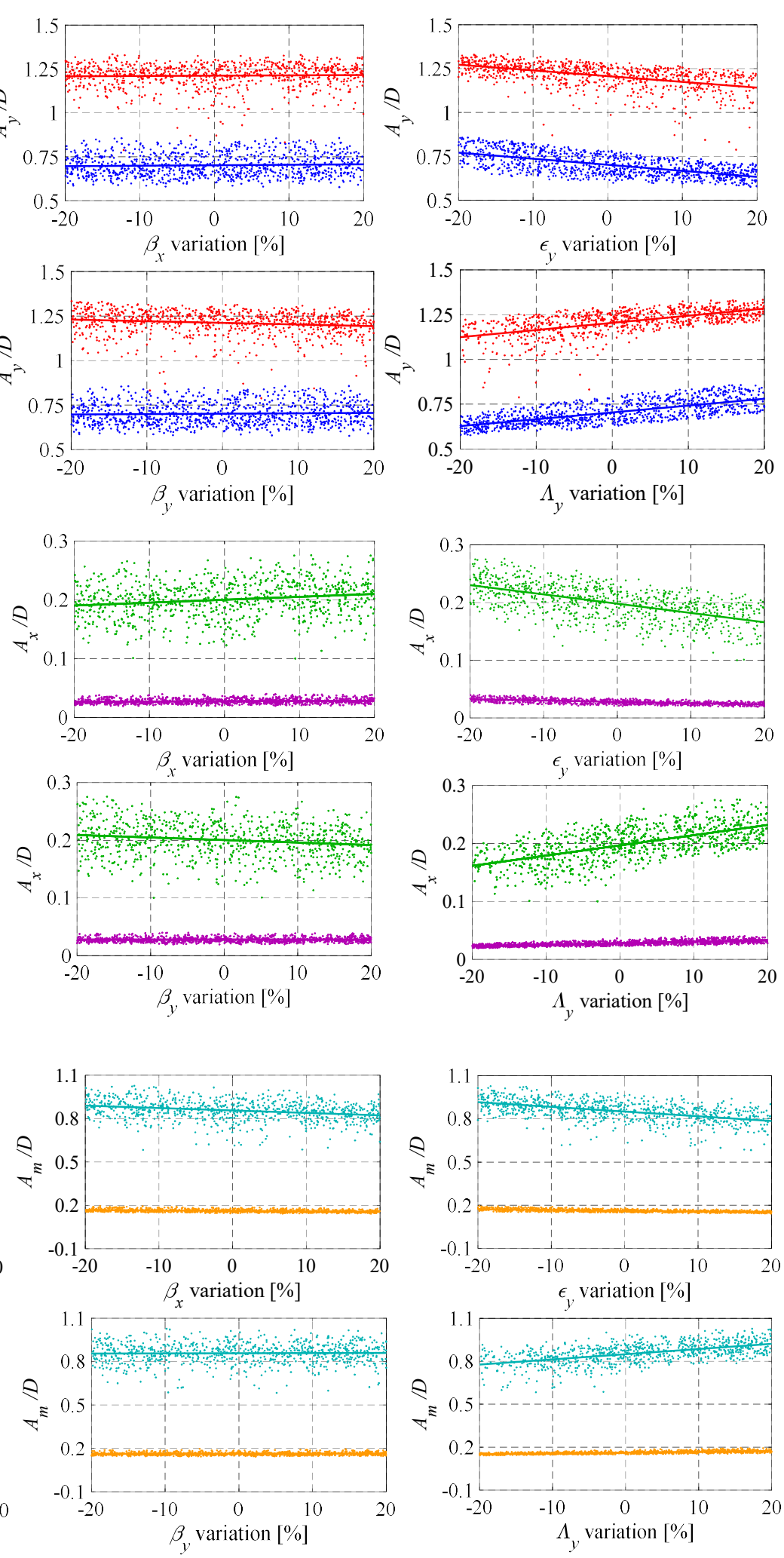

Figure 7 Scatterplots and least-square fitting lines of response outputs vs. six empirical input variations: red, green and turquoise (blue, purple and orange) maps of $A_{y} / D, A_{x} / D$ and $A_{m} / D$, respectively, denote $m^{*}=3.68$ and $V_{r}=9\left(m^{*}=8.76\right.$ and $\left.V_{r}=6\right)$. 
a)
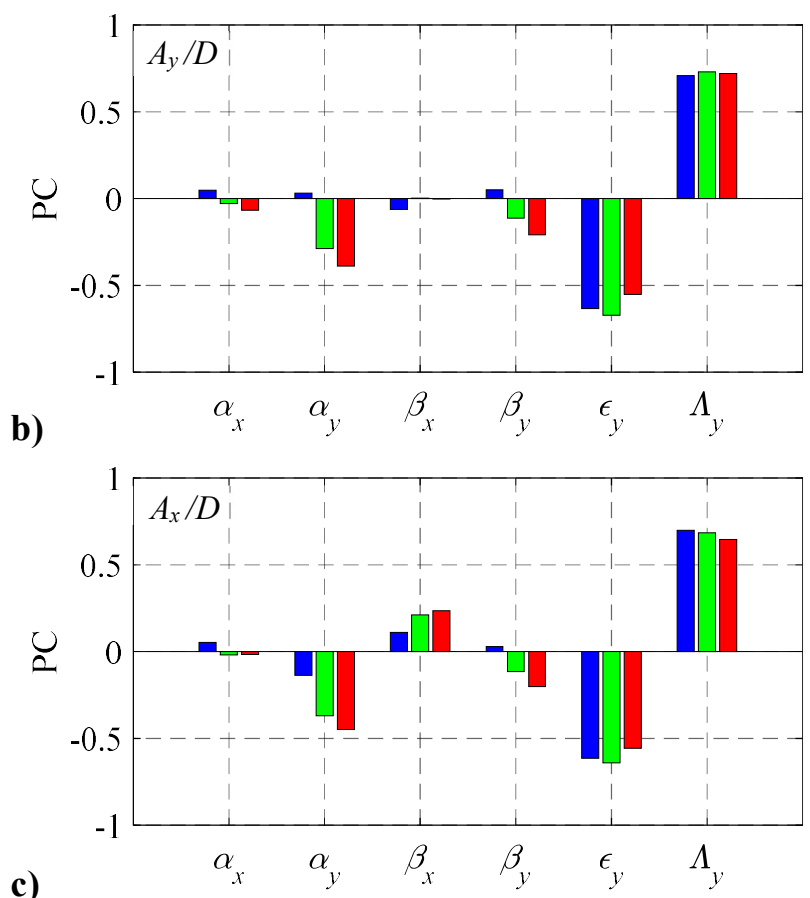

c)

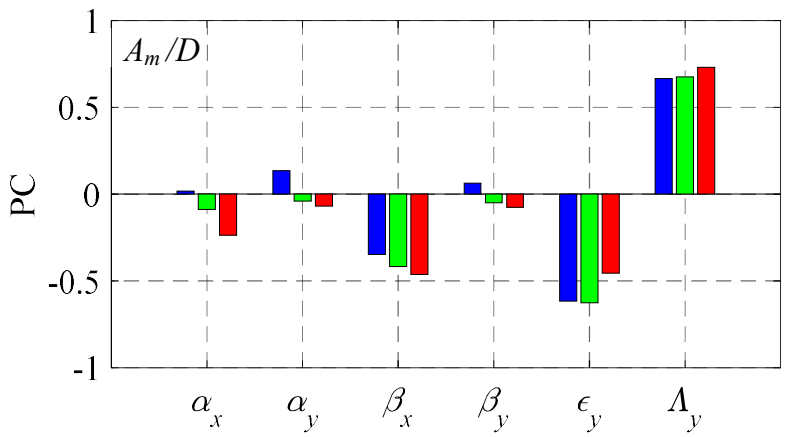

Figure 8 Pearson's input-output correlation coefficients at $V_{r}=6$ : red, green and blue bars denote $m^{*}=3.68,5.19$ and 8.76 , respectively. 
a)
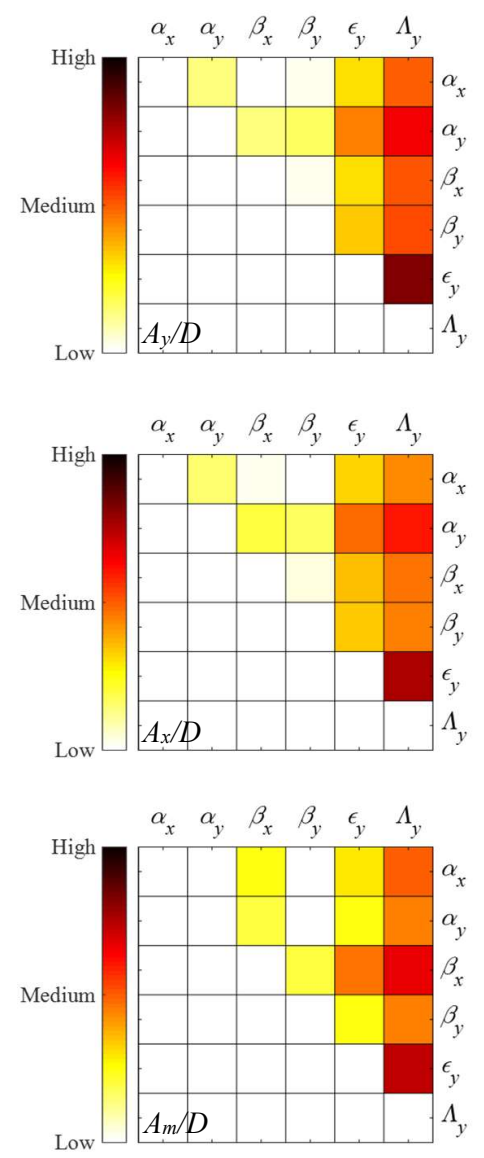

b)
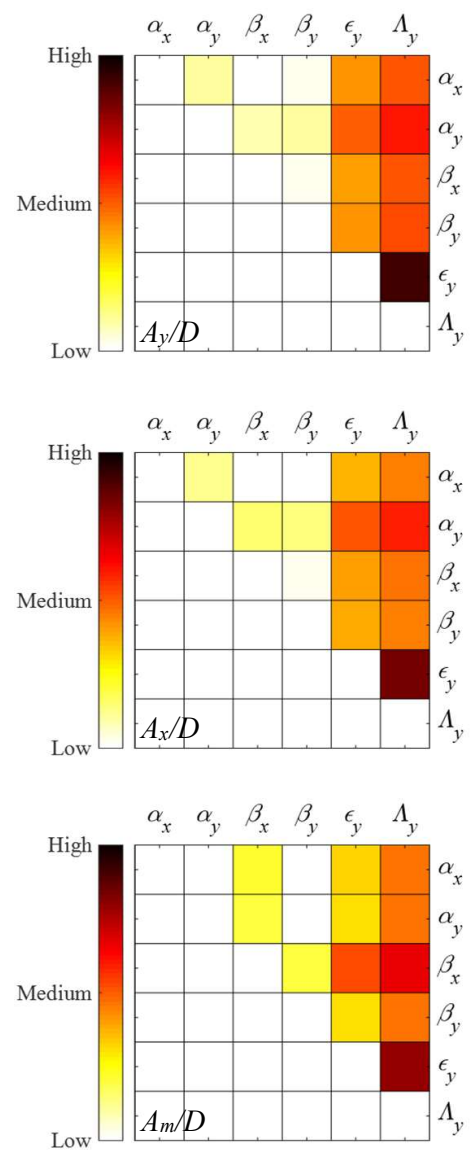

c)
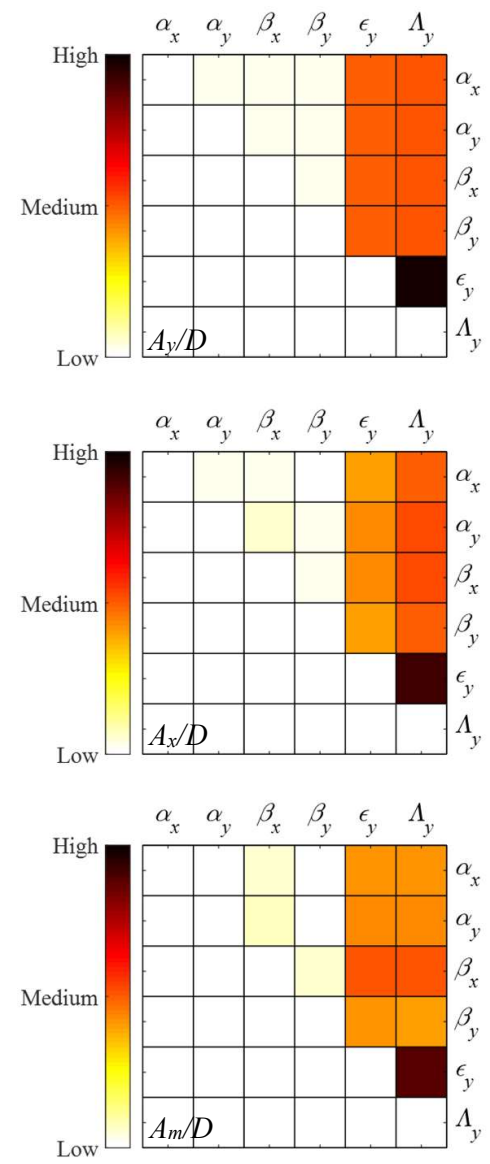

Figure 9 Second-order Sobol sensitivity indices for different inputs versus outputs: columns a), b) and c) represent results with $m^{*}=3.68,5.19$ and 8.76 , respectively. 
a)

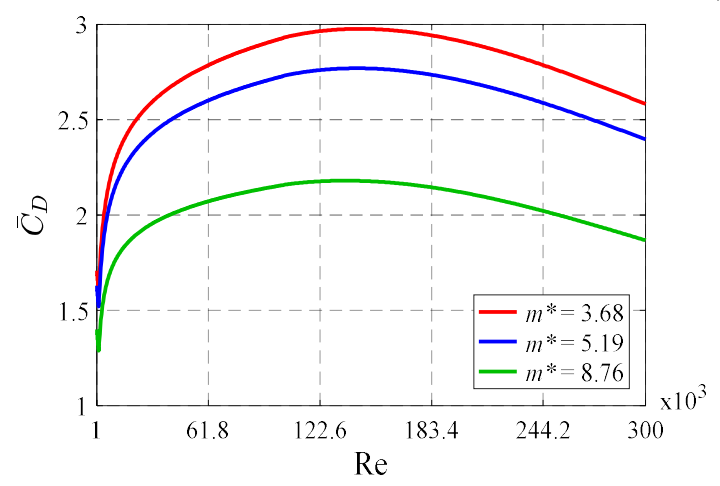

c)
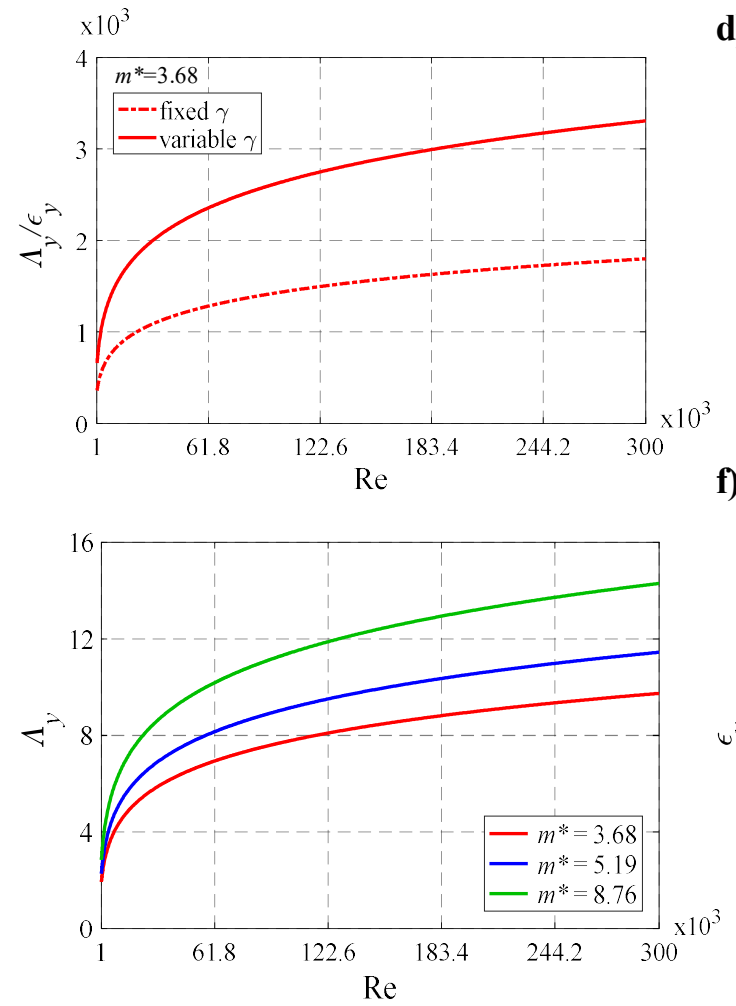

b)

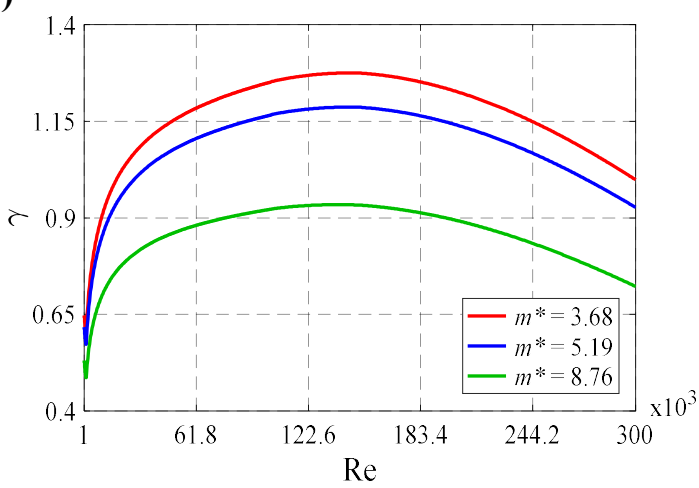

d)
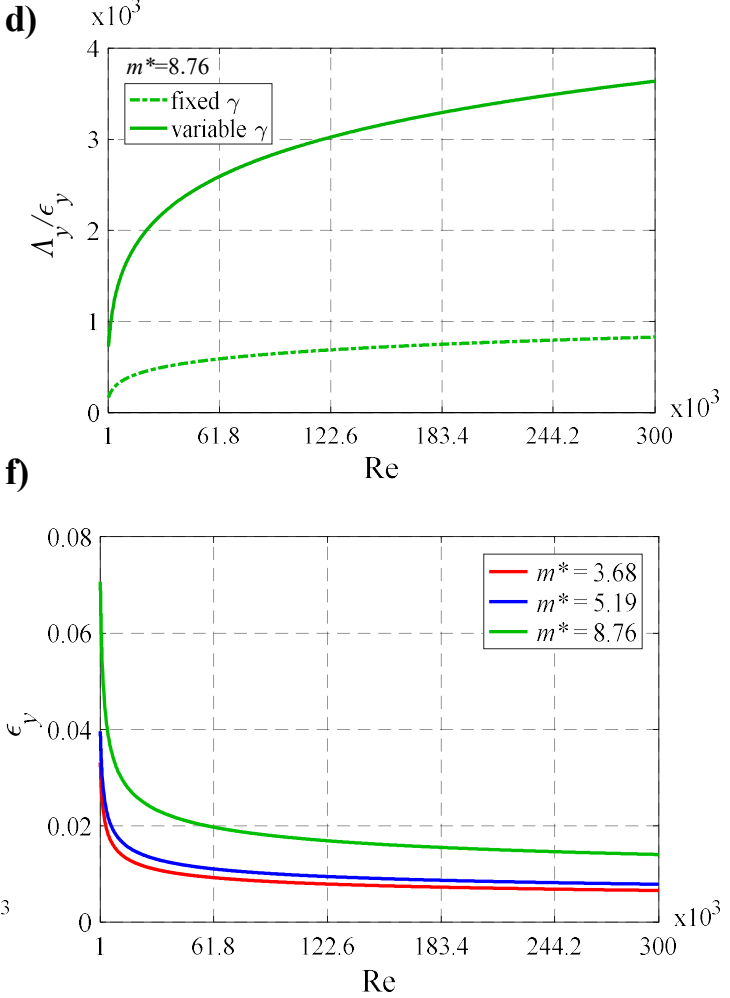

Figure 10 Variation of hydrodynamic (a, b) and empirical coefficients (c-f) as function of Re for cylinder with different $m^{*}$ : (a) amplification of $\bar{C}_{D}$ based on Eqs. (13) and (33), (b) $\gamma=\bar{C}_{D} / 4 \pi \mathrm{St}$ based on Figs. 3a and $10 \mathrm{a}, \Lambda_{y} / \varepsilon_{y}$ in (c) and (d) based on Eq. (34) with variable $\gamma$ or fixed $\gamma=0.8$, (e) $\Lambda_{y}$ variation with fixed $\varepsilon_{y}$ through Eq. (14), (f) $\varepsilon_{y}$ variation with fixed $\Lambda_{y}=12$. 
a)
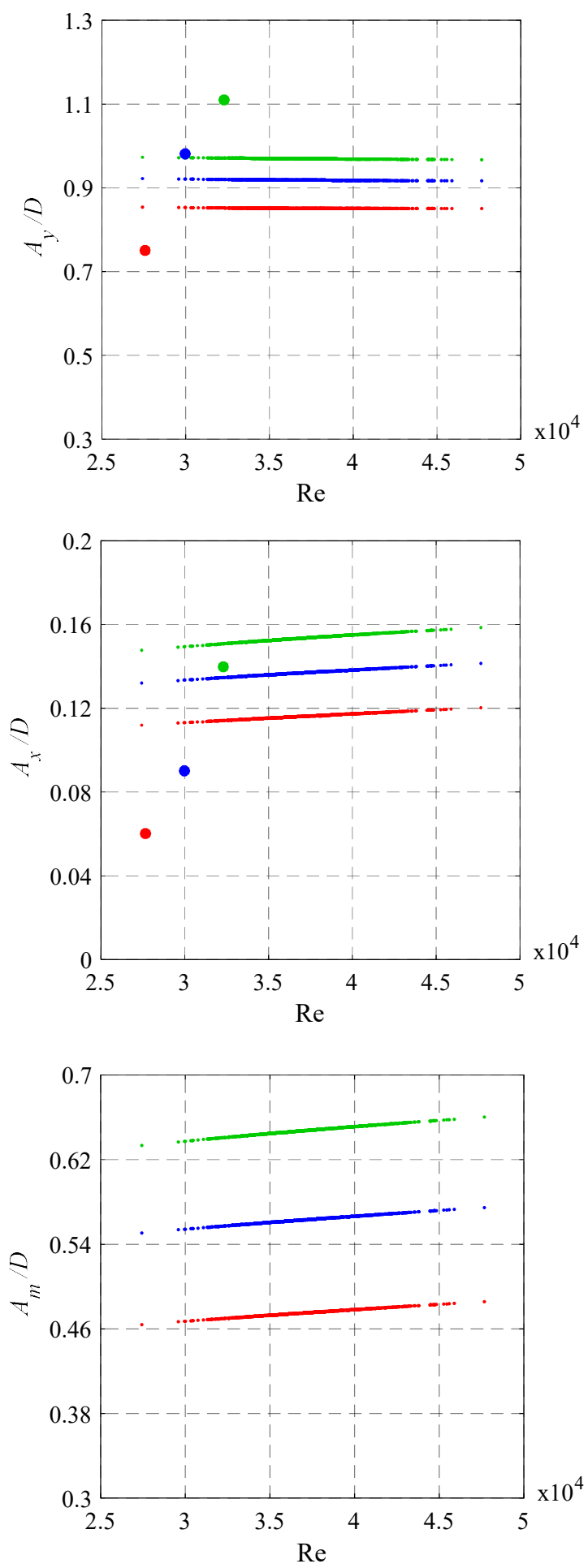

b)
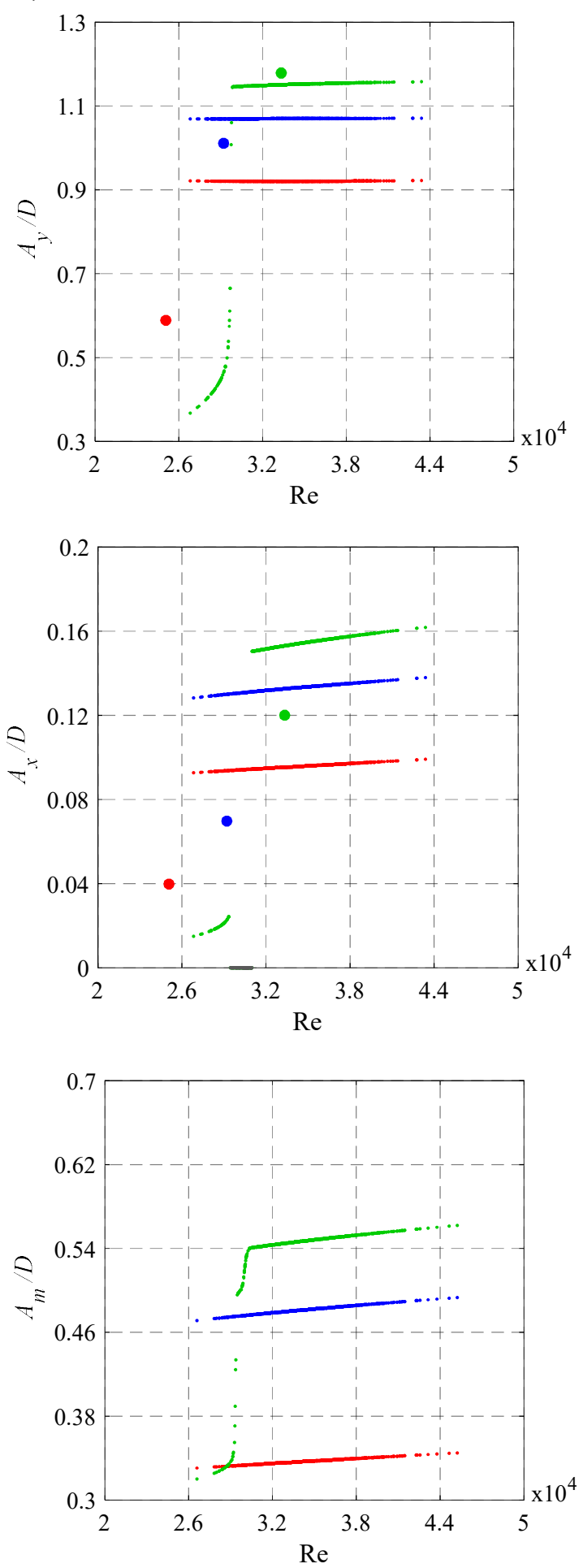

Figure 11 Sensitivity to Re of maximum $A_{y} / D, A_{x} / D$ and $A_{m} / D$ with $V_{r}=6$ (red), 6.5 (blue) and 7 (green): (a) $m^{*}=3.68$, (b) $m^{*}=5.19$; circles denote test data at the same Re and $V_{r}$ (Stappenbelt et al., 2007). 


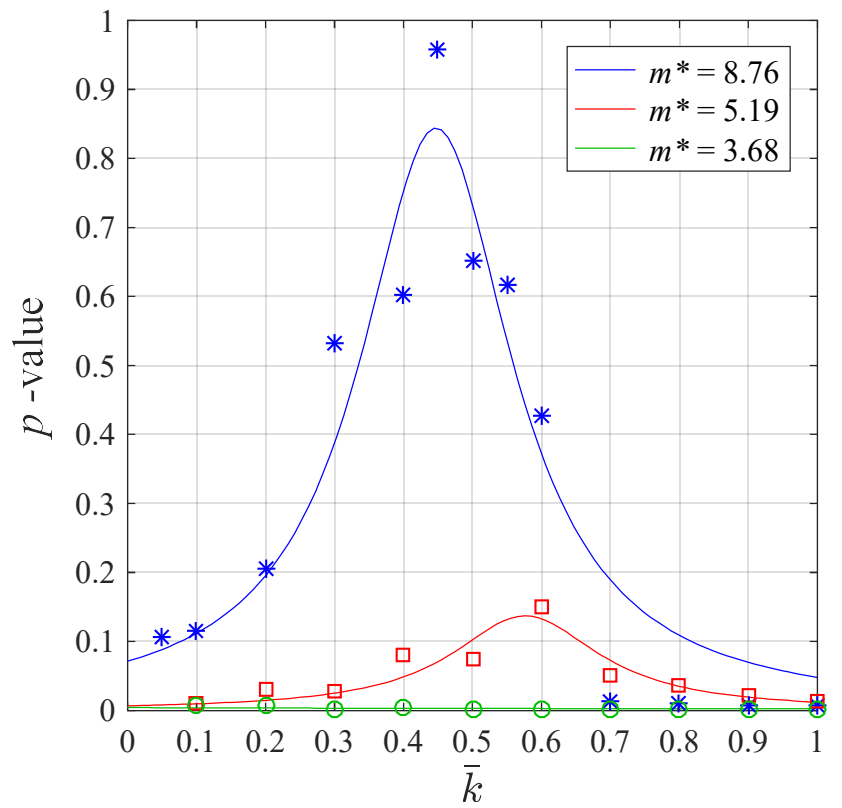

Figure 12 Statistical $p$-values (symbols) as a function of the amplified drag coefficient $\bar{k}$ in Eq. (13) for $m^{*}=3.68$ (green), 5.19 (red) and 8.76 (blue): solid lines denote the associated curve-fitting trends. 

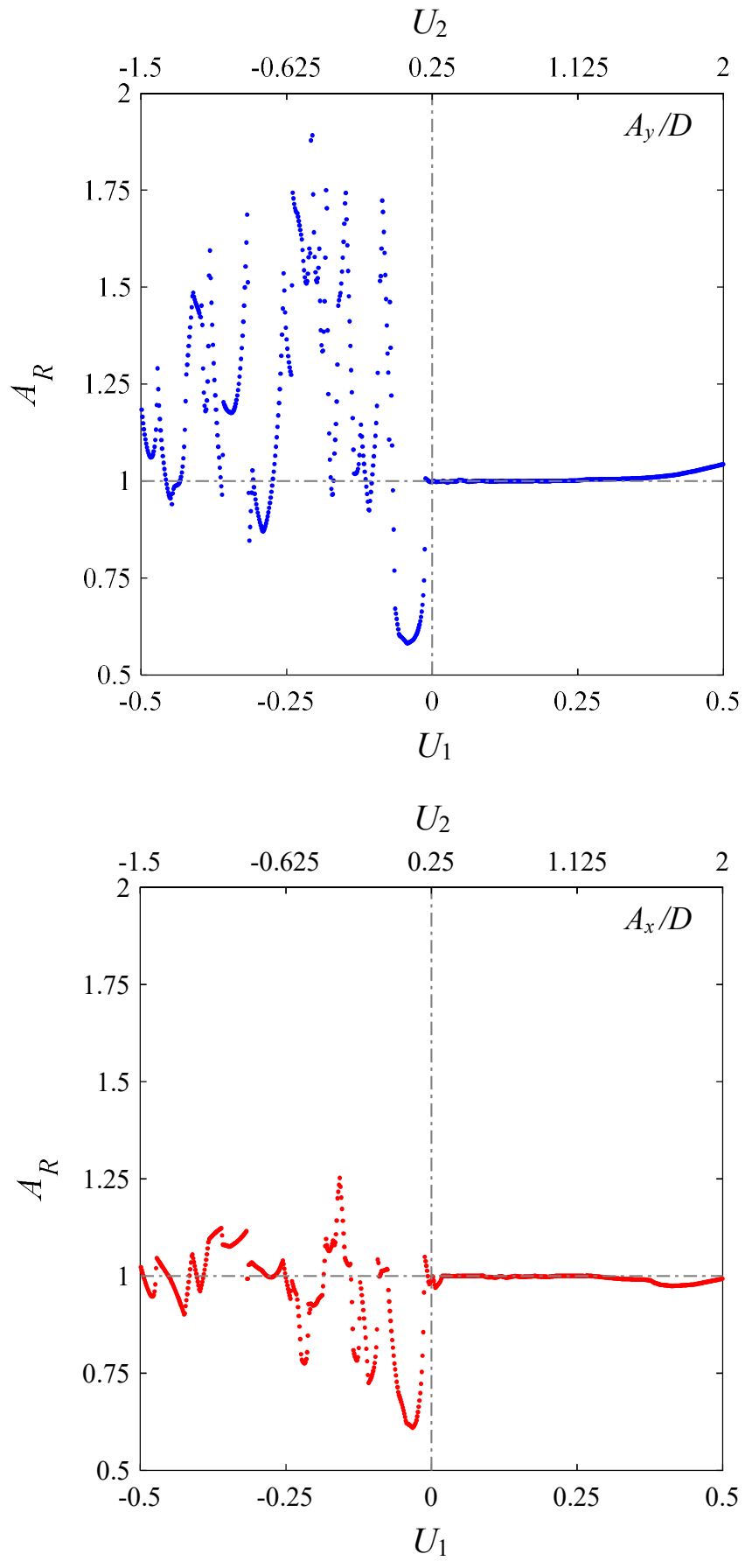

Figure 13 Variation of response amplitude ratios $A_{R}$ between correlated and uncorrected variable models vs. the normal space $U_{1}$ and $U_{2}$ parameters associated with interdependent $\bar{C}_{D}$ and $A^{*}$, respectively. 
Table 1 Model parameters and hydrodynamic coefficients.

\begin{tabular}{|l|l|l|}
\hline Dimensionless quantities & Hydrodynamic coefficients & Fluid-structure parameters \\
\hline$M_{L}$ (Eq. 9) & $C_{L 0}, \mathrm{St}, C_{a}$ & $m^{*}$ \\
\hline$M_{D}$ & $C_{D 0}, \mathrm{St}, C_{a}$ & $m^{*}$ \\
\hline $\bar{M}_{D}$ & $\bar{C}_{D}, \mathrm{St}, C_{a}$ & $m^{*}$ \\
\hline$\lambda$ (Eq. 11) & $\bar{C}_{D}, C_{a}$ & $m^{*}, \xi_{x}, V_{r}$ \\
\hline$\Omega$ & $\mathrm{St}$ & $V_{r}$ \\
\hline
\end{tabular}

Table 2 Empirical wake-cylinder and hydrodynamic coefficients as mean values in Monte Carlo Simulations

\begin{tabular}{|c|c|c|c|c|c|}
\hline $\begin{array}{c}\text { Wake } \\
\text { coefficients }\end{array}$ & Values & $\begin{array}{c}\text { Cylinder } \\
\text { coefficients }\end{array}$ & Values & $\begin{array}{c}\text { Hydrodynamic } \\
\text { coefficients }\end{array}$ & Values \\
\hline$\varepsilon_{x}$ & 0.3 & $\alpha_{x}$ & 0.7 & $C_{L 0}$ & 0.3 \\
\hline$\Lambda_{x}$ & 12 & $\beta_{x}$ & 0.7 & $C_{D 0}$ & 0.2 \\
\hline$\varepsilon_{y}$ & Eq. (14) & $\alpha_{y}$ & 0.7 & $\bar{C}_{D}$ & 2 \\
\hline$\Lambda_{y}$ & 12 & $\beta_{y}$ & 0.7 & $\mathrm{St}$ & 0.2 \\
\hline
\end{tabular}


Table 3 Coefficient of determinations $\left(\mathrm{R}^{2}\right)$ and absolute standardised regression coefficients (SRC) with ranking of the relative importance for $A_{y} / D$ outputs: top, middle and bottom rows denote the cases of $m^{*}=$ $3.68,5.19$ and 8.76, respectively, with underlined maximum SRC.

\begin{tabular}{|c|cccccccc|c|}
\hline Regression & \multicolumn{7}{|c|}{$\boldsymbol{V}_{\boldsymbol{r}}$} & \\
\cline { 2 - 9 } \\
analysis & $\mathbf{4}$ & $\mathbf{5}$ & $\mathbf{6}$ & $\mathbf{7}$ & $\mathbf{8}$ & $\mathbf{9}$ & $\mathbf{1 0}$ & Rank \\
\hline $\mathrm{R}^{2}$ & 0.997 & 0.998 & 0.997 & 0.990 & 0.942 & 0.789 & 0.671 & \\
& 0.997 & 0.998 & 0.996 & 0.953 & 0.780 & & & \\
& 0.881 & 0.996 & 0.947 & 0.772 & & & & \\
\hline $\operatorname{SRC}\left(\alpha_{x}\right)$ & 0.001 & 0.001 & 0.001 & 0.002 & 0.015 & 0.023 & $\underline{0.053}$ & $6^{\text {th }}$ \\
& 0.003 & 0.002 & 0.001 & 0.001 & $\underline{0.020}$ & & & \\
& 0.005 & 0.000 & 0.001 & $\underline{0.009}$ & & & & \\
\hline $\operatorname{SRC}\left(\alpha_{y}\right)$ & 0.234 & $\underline{0.453}$ & 0.382 & 0.250 & 0.070 & 0.035 & 0.167 & $3^{\text {rd }}$ \\
& 0.151 & $\underline{0.417}$ & 0.276 & 0.042 & 0.189 & & & \\
& 0.044 & $\underline{0.296}$ & 0.035 & 0.139 & & & & \\
\hline $\operatorname{SRC}\left(\beta_{x}\right)$ & 0.005 & 0.036 & 0.027 & 0.008 & 0.044 & 0.034 & $\underline{0.057}$ & $5^{\text {th }}$ \\
& 0.004 & 0.019 & 0.019 & 0.014 & $\underline{0.024}$ & & & \\
& $\underline{0.019}$ & 0.005 & 0.002 & 0.018 & & & & \\
\hline $\operatorname{SRC}\left(\beta_{y}\right)$ & 0.037 & 0.096 & 0.166 & $\underline{0.210}$ & 0.169 & 0.101 & 0.047 & $4^{\text {th }}$ \\
& 0.018 & 0.048 & $\underline{0.066}$ & 0.048 & 0.041 & & & \\
& 0.002 & 0.020 & 0.003 & $\underline{0.034}$ & & & & \\
\hline $\operatorname{SRC}\left(\varepsilon_{y}\right)$ & 0.183 & 0.391 & 0.548 & 0.626 & 0.638 & 0.667 & $\underline{0.863}$ & $2^{\text {nd }}$ \\
& 0.134 & 0.452 & 0.622 & $\underline{0.652}$ & 0.390 & & & \\
& 0.076 & 0.615 & $\underline{0.666}$ & 0.230 & & & & \\
\hline $\operatorname{SRC}\left(\Lambda_{y}\right)$ & 0.948 & 0.783 & 0.708 & 0.682 & 0.691 & 0.790 & $\underline{0.969}$ & $1^{\text {st }}$ \\
& $\underline{0.964}$ & 0.742 & 0.673 & 0.671 & 0.744 & & & \\
& $\underline{0.936}$ & 0.757 & 0.740 & 0.845 & & & & \\
\hline
\end{tabular}

Table 4 Coefficient of determinations $\left(\mathrm{R}^{2}\right)$ and absolute standardised regression coefficients (SRC) with ranking of the relative importance for $A_{x} / D$ outputs: top, middle and bottom rows denote the cases of $m^{*}=$ 3.68, 5.19 and 8.76, respectively, with underlined maximum SRC.

\begin{tabular}{|c|ccccccc|c|}
\hline Regression & \multicolumn{7}{|c|}{$\boldsymbol{V}_{\boldsymbol{r}}$} & \multicolumn{1}{c|}{ Rank } \\
\cline { 2 - 9 } analysis & $\mathbf{4}$ & $\mathbf{5}$ & $\mathbf{6}$ & $\mathbf{7}$ & $\mathbf{8}$ & $\mathbf{9}$ & $\mathbf{1 0}$ & Rank \\
\hline $\mathrm{R}^{2}$ & 0.994 & 0.992 & 0.993 & 0.992 & 0.980 & 0.950 & 0.864 & \\
& 0.984 & 0.990 & 0.990 & 0.980 & 0.831 & & & \\
& 0.803 & 0.989 & 0.959 & 0.749 & & & & \\
\hline $\mathrm{SRC}\left(\alpha_{x}\right)$ & 0.010 & 0.027 & 0.052 & $\underline{0.065}$ & 0.050 & 0.027 & 0.004 & $6^{\text {th }}$ \\
& 0.011 & 0.019 & $\underline{0.032}$ & 0.031 & 0.010 & & & \\
& $\underline{0.012}$ & 0.007 & 0.002 & 0.011 & & & & \\
\hline $\operatorname{SRC}\left(\alpha_{y}\right)$ & 0.240 & $\underline{0.481}$ & 0.431 & 0.311 & 0.147 & 0.028 & 0.071 & $3^{\text {rd }}$ \\
& 0.160 & $\underline{0.437}$ & 0.362 & 0.151 & 0.165 & & & \\
& 0.054 & $\underline{0.268}$ & 0.133 & 0.147 & & & & \\
\hline $\operatorname{SRC}\left(\beta_{x}\right)$ & 0.058 & 0.162 & 0.208 & $\underline{0.221}$ & 0.204 & 0.186 & 0.180 & $4^{\text {th }}$ \\
& 0.040 & 0.161 & $\underline{0.197}$ & 0.178 & 0.016 & & & \\
& 0.013 & 0.166 & $\underline{0.175}$ & 0.004 & & & & \\
\hline $\operatorname{SRC}\left(\beta_{y}\right)$ & 0.039 & 0.101 & 0.162 & $\underline{0.190}$ & 0.158 & 0.111 & 0.056 & $5^{\text {th }}$ \\
& 0.019 & 0.048 & $\underline{0.073}$ & 0.063 & 0.027 & & & \\
& 0.005 & 0.015 & 0.011 & $\underline{0.026}$ & & & & \\
\hline $\operatorname{SRC}\left(\varepsilon_{y}\right)$ & 0.244 & 0.423 & 0.538 & 0.607 & 0.639 & 0.732 & $\underline{0.896}$ & $2^{\text {nd }}$ \\
& 0.182 & 0.464 & 0.595 & $\underline{0.645}$ & 0.445 & & & \\
& 0.094 & 0.623 & $\underline{0.659}$ & 0.274 & & & & \\
\hline $\operatorname{SRC}\left(\Lambda_{y}\right)$ & 0.930 & 0.724 & 0.647 & 0.641 & 0.679 & 0.830 & $\underline{0.966}$ & $1^{\text {st }}$ \\
& 0.942 & 0.697 & 0.621 & 0.653 & 0.747 & & & \\
& $\underline{0.892}$ & 0.751 & 0.737 & 0.820 & & & & \\
\hline
\end{tabular}


Table 5 Coefficient of determinations $\left(\mathrm{R}^{2}\right)$ and absolute standardised regression coefficients (SRC) with ranking of the relative importance for $A_{m} / D$ outputs: top, middle and bottom rows denote the cases of $m^{*}=$ $3.68,5.19$ and 8.76, respectively, with underlined maximum SRC.

\begin{tabular}{|c|c|c|c|c|c|c|c|c|}
\hline \multirow{2}{*}{$\begin{array}{c}\text { Regression } \\
\text { analysis }\end{array}$} & \multicolumn{7}{|c|}{$V_{r}$} & \multirow[b]{2}{*}{ Rank } \\
\hline & 4 & 5 & 6 & 7 & 8 & 9 & 10 & \\
\hline \multirow[t]{3}{*}{$\mathrm{R}^{2}$} & 0.985 & 0.994 & 0.995 & 0.996 & 0.988 & \multirow[t]{3}{*}{0.913} & \multirow[t]{3}{*}{0.853} & \\
\hline & 0.979 & 0.992 & 0.993 & 0.986 & 0.853 & & & \\
\hline & 0.760 & 0.987 & 0.965 & 0.732 & & & & \\
\hline \multirow[t]{3}{*}{$\operatorname{SRC}\left(\alpha_{x}\right)$} & 0.029 & 0.094 & 0.188 & 0.263 & 0.296 & \multirow[t]{3}{*}{0.308} & \multirow[t]{3}{*}{$\underline{0.391}$} & \multirow[t]{3}{*}{$4^{\text {th }}$} \\
\hline & 0.008 & 0.050 & 0.100 & 0.120 & 0.063 & & & \\
\hline & 0.002 & 0.021 & $\underline{0.027}$ & $\overline{0.003}$ & & & & \\
\hline \multirow[t]{3}{*}{$\operatorname{SRC}\left(\alpha_{y}\right)$} & 0.207 & 0.228 & 0.090 & 0.022 & 0.112 & \multirow[t]{3}{*}{0.173} & \multirow[t]{3}{*}{$\underline{0.293}$} & \multirow[t]{3}{*}{$5^{\text {th }}$} \\
\hline & 0.149 & 0.207 & 0.022 & 0.134 & 0.224 & & & \\
\hline & 0.054 & 0.113 & 0.138 & 0.177 & & & & \\
\hline \multirow[t]{3}{*}{$\operatorname{SRC}\left(\beta_{x}\right)$} & 0.134 & 0.394 & $\underline{0.476}$ & 0.444 & 0.343 & \multirow[t]{3}{*}{0.267} & \multirow[t]{3}{*}{0.216} & \multirow[t]{3}{*}{$3^{\text {rd }}$} \\
\hline & 0.081 & 0.369 & 0.449 & 0.358 & 0.100 & & & \\
\hline & 0.052 & 0.302 & $\overline{0.286}$ & 0.084 & & & & \\
\hline \multirow[t]{3}{*}{$\operatorname{SRC}\left(\beta_{y}\right)$} & 0.037 & 0.050 & 0.042 & 0.019 & 0.021 & \multirow[t]{3}{*}{0.070} & \multirow[t]{3}{*}{$\underline{0.138}$} & \multirow[t]{3}{*}{$6^{\text {th }}$} \\
\hline & 0.018 & 0.023 & 0.010 & 0.015 & $\underline{0.053}$ & & & \\
\hline & 0.008 & 0.012 & 0.008 & $\underline{0.018}$ & & & & \\
\hline \multirow[t]{3}{*}{$\operatorname{SRC}\left(\varepsilon_{y}\right)$} & 0.134 & 0.329 & 0.476 & $\overline{0.558}$ & 0.596 & \multirow[t]{3}{*}{0.676} & \multirow[t]{3}{*}{$\underline{0.877}$} & \multirow[t]{3}{*}{$2^{\text {nd }}$} \\
\hline & 0.109 & 0.422 & 0.576 & 0.633 & 0.454 & & & \\
\hline & 0.059 & 0.580 & 0.633 & $\overline{0.283}$ & & & & \\
\hline \multirow[t]{3}{*}{$\operatorname{SRC}\left(\Lambda_{y}\right)$} & 0.941 & 0.803 & $\overline{0.690}$ & 0.626 & 0.617 & \multirow[t]{3}{*}{0.743} & \multirow[t]{3}{*}{$\underline{0.958}$} & \multirow[t]{3}{*}{$1^{\text {st }}$} \\
\hline & $\underline{0.958}$ & 0.770 & 0.644 & 0.623 & 0.737 & & & \\
\hline & $\overline{0.865}$ & 0.742 & 0.686 & 0.792 & & & & \\
\hline
\end{tabular}




\section{Highlights}

- Monte Carlo-based sensitivity analyses of nonlinear wake-cylinder oscillators are presented.

- Contribution, relative importance, coupling and interdependence of empirical variables are studied.

- Reynolds number dependence of empirical wake and hydrodynamic coefficients is accounted for.

- Most influential variables correspond to lift force wake oscillator governing cross-flow/in-line VIV.

- Input sensitivities and output uncertainties depend on system parameters and response amplitudes. 